Article

\title{
Comparative Transcriptome Analysis of Gene Expression and Regulatory Characteristics Associated with Different Vernalization Periods in Brassica rapa
}

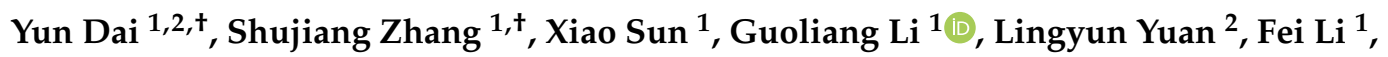 \\ Hui Zhang ${ }^{1}$, Shifan Zhang ${ }^{1}$, Guohu Chen ${ }^{2}$, Chenggang Wang ${ }^{2, *(D)}$ and Rifei Sun ${ }^{1, *(D)}$ \\ 1 Institute of Vegetables and Flowers, Chinese Academy of Agricultural Sciences, Beijing 100081, China; \\ dy1027@ahau.edu.cn (Y.D.); zhangshujiang@caas.cn (S.Z.); 13121273253@163.com (X.S.); \\ liguoliang@caas.cn (G.L.); lifei@caas.cn (F.L.); zhanghui05@caas.cn (H.Z.); zhangshifan@caas.cn (S.Z.) \\ 2 Vegetable Genetics and Breeding Laboratory, College of Horticulture, Anhui Agricultural University, \\ Hefei 230036, China; yuanlingyun@ahau.edu.cn (L.Y.); cgh@ahau.edu.cn (G.C.) \\ * Correspondence: cgwang@ahau.edu.cn (C.W.); sunrifei@caas.cn (R.S.) \\ + Equal contributors.
}

Received: 22 February 2020; Accepted: 3 April 2020; Published: 5 April 2020

\begin{abstract}
Brassica rapa is an important Chinese vegetable crop that is beneficial to human health. The primary factor affecting $B$. rapa yield is low temperature, which promotes bolting and flowering, thereby lowering its commercial value. However, quickened bolting and flowering can be used for rapid breeding. Therefore, studying the underlying molecular mechanism of vernalization in $B$. rapa is crucial for solving production-related problems. Here, the transcriptome of two $B$. rapa accessions were comprehensively analyzed during different vernalization periods. During vernalization, a total of $974,584,022$ clean reads and $291.28 \mathrm{~Gb}$ of clean data were obtained. Compared to the reference genome of $B$. rapa, 44,799 known genes and 2280 new genes were identified. A self-organizing feature map analysis of 21,035 differentially expressed genes was screened in two B. rapa accessions, 'Jin Wawa' and 'Xiao Baojian'. The analysis indicated that transcripts related to the plant hormone signal transduction, starch and sucrose metabolism, photoperiod and circadian clock, and vernalization pathways changed notably at different vernalization periods. Moreover, different expression patterns of TPS, UGP, CDF, VIN1, and seven hormone pathway genes were observed during vernalization between the two accessions. The transcriptome results of this study provide a new perspective on the changes that occur during B. rapa vernalization, as well as serve as an excellent reference for B. rapa breeding.
\end{abstract}

Keywords: Brassica rapa; enrichment pathway; self-organizing feature map; transcriptome analysis; vernalization

\section{Introduction}

The vegetative and reproductive growth stage are important stages in the life cycle of land plants. After a period of vegetative growth, land plants undergo reproductive growth, which is regulated by various environmental factors and endogenous signals that induce flowering. This transformation process is called floral induction, also known as flowering transition. A regulatory network ensures that plants accumulate sufficient vegetative growth over time, transforming them into reproductive growth [1]. With the development of molecular biology, five pathways that influence bolting and flowering in rice, wheat, and Arabidopsis have been identified, including the photoperiod, gibberellin (GA), vernalization, autonomous, and age pathways [2-6]. 
Typically, vernalization and long periods of daylight are the main factors that drive plant vegetative growth to reproductive development $[7,8]$. Thus far, vernalization and long periods of daylight have been found to regulate flowering factors, including the flowering promoter $(F T)$ and related interaction genes that affect the flowering time [9]. In Arabidopsis thaliana, the vernalization pathway molecular mechanism regulates flower locus C (FLC), frigida (FRI), vernalization 1 (VRN1), vernalization 2 (VRN2), and vernalization insensitive 3 (VIN3). FRI promotes the high expression of FLC and the low expression of $F T$, which leads to flowering delays [10]. VRN1, VRN2, and VIN3 are negative regulators of $F L C$, of which, VRN2 was the first vernalization gene to be cloned [11]. VRN1 and $V R N 2$ are expressed in different plant tissues and developmental stages. They are not induced by vernalization, but control the low expression of $F L C$ after vernalization has concluded [12,13]. VIN3 is induced by vernalization, participates in FLC chromatin remodeling, and plays a suppressive role [14]. In monocotyledonous wheat, the genes necessary for vernalization include $V R N 1, V R N 2$, and vernalization 3 (VRN3), none of which are homologues to the VRNs of Arabidopsis and do not contain FLC-like $[15,16]$. Both VRN1 and VRN3 in Arabidopsis have homology genes FUL and FT in wheat $[15,17]$. VRN2 possesses no homologous genes in Arabidopsis and is a negative regulator of flowering in wheat during unvernalized [18]. Differences in the vernalization pathways between Arabidopsis and monocotyledons may be due to the independent evolution of their response mechanisms to vernalization.

Brassica rapa is a leafy vegetable that belongs to the Brassicaceae family. It originated in China and has been widely distributed throughout the country, accounting for a wide area of vegetable cultivation. B. rapa is a high-yielding vegetable, is durable to storage and transportation, possesses a long supply period, is rich in nutrition, and diverse in consumption methods. This plant also occupies the primary position in vegetable production and daily consumption in China. It is generally planted in the fall from August to November. Spring planting was later developed to meet the demand for supply throughout the year. Spring sowing occurs under low temperature conditions and periods of long daylight, which cause B. rapa to prematurely bolt and flower, thereby causing the plant to lose some of its commercial value [18]. However, quickened bolting is a rapid breeding method that can help produce better accessions faster. Therefore, low-temperature vernalization has some positive effects on the cultivation of $B$. rapa, but further research is required to verify these effects.

In this study, a 50-day vernalization experiment was conducted using two B. rapa accessions. Samples with different vernalization times were selected according to their flowering times for transcriptome analysis. The expression of differentially expressed genes (DEGs) in the plant hormone signal transduction, starch and sucrose metabolism, photoperiod and circadian clock, and vernalization pathways were analyzed during two vernalization processes. The purpose of this study was to clarify the effects of different vernalization periods on the two different bolting B. rapa accessions.

\section{Materials and Methods}

\subsection{Plant Materials andTreatments}

Two B. rapa accessions, 200 late-bolting 'Jin Wawa' (JWW) and 200 early-bolting 'Xiao Baojian' $(\mathrm{XBJ})$, were grown in a nursery greenhouse at the Chinese Academy of Agricultural Sciences in Beijing, China $\left(39^{\circ} 56^{\prime} \mathrm{N}, 116^{\circ} 20^{\prime} \mathrm{E}\right)$, which consisted of highly inbred lines. Plants were initially grown at $25 \pm 2{ }^{\circ} \mathrm{C}$ under natural light for 32 days. Afterwards, 100 'JWW' and 100 'XBJ' were moved into a vernalization room at $4{ }^{\circ} \mathrm{C}$ under a $16 / 8 \mathrm{~h}$ light/dark photoperiod and $150 \mu \mathrm{mol} \mathrm{m}{ }^{-2} \mathrm{~s}^{-1}$ light intensity. Plants were randomly arranged. The other 100 'JWW' and 100 ' $\mathrm{XBJ}^{\circ}$ were moved into a climate chamber at $25^{\circ} \mathrm{C}$ under the same conditions as the vernalization room. The day when two B. rapa accessions transferred into the vernalization room and the climate chamber were recorded as 0days, and the vernalization and control were treated for a total of 50 days, with each 5 days being considered a period. For the time of day after treatment (DAT), the default DAT conditions were $4{ }^{\circ} \mathrm{C}$ for the vernalization treatment days and $25^{\circ} \mathrm{C}$ DAT for the control treatment days. The third fully expanded 
leaves from the center of the plants were sampled every 5 days from both treatments for a total of 50 days. Three biological replicates of the leaves were randomly collected from each individual seedling. Each treatment sample was collected separately, immediately frozen in liquid nitrogen, and stored at $-80{ }^{\circ} \mathrm{C}$ for future transcriptome sequencing (i.e., RNA-Seq) and quantitative real-time polymerase chain reaction (qRT-PCR) analyses. After random sampling during each period, eight seedlings from the previous $4{ }^{\circ} \mathrm{C}$ treatment and grown in a nursery greenhouse $\left(25 \pm 2{ }^{\circ} \mathrm{C}\right.$ under natural light). The flowering times were recorded, which began when the first petal was fully expanded.

\subsection{RNA Extraction and Illumina Sequencing}

Total RNA was extracted from leaf samples using an RNAprep Pure Plant kit (Tiangen, Beijing, China) following the manufacturer's instructions. RNA concentrations and quality were verified using an Agilent 2100 Bioanalyzer (Agilent Technologies, Santa Clara, CA, USA). A total amount of $1 \mu \mathrm{g}$ RNA per sample was used as input material for the RNA sample preparations. Forty-two cDNA libraries (2 accessions $\times 7$ periods $\times 3$ replicates) were constructed and sequenced using NEBNext $\left(\right.$ Ultra ${ }^{\mathrm{TM}}$ RNA Library Prep Kit for Illumina (New England Biolabs, Ipswich, MA, USA) following manufacturer's recommendations. mRNA was purified from total RNA using poly-T oligo-attached magnetic beads. cDNA fragments of preferentially $240 \mathrm{bp}$ in length were selected, the library fragments were purified with AMPure XP system (Beckman Coulter, Brea, CA, USA).Each library generated $>6$ GB raw data. Raw reads were processed in fastq format. The adaptor sequences and low-quality sequence reads were removed from the data sets, including reads that removed more than $10 \%$ of $\mathrm{N}$ and more than $50 \%$ of reads with a base of $\mathrm{Q} \leq 10$. The raw data have been submitted under BioProject number PRJNA615255 to the Sequence Read Archive (SRA) database at NCBI.

\subsection{Transcriptome Analysis}

The B. rapa reference genome sequence and annotation files were downloaded from the Brassica Database v3.0 [19]. Clean reads were mapped to the B. rapa reference genome sequence using Tophat v2.0.9 [20]. Cufflinks v2.2.1 was used to assemble reference annotation files, estimate the levels of unigene expression, and normalize the relative abundance of each transcript in each sample to fragments per kilobase of exon per million fragments mapped (FPKM) [21]. DESeq R v1.10.1 was used for processing to detect transcriptional changes in 'JWW' and ' $\mathrm{XBJ}$ ' during vernalization [22]. DEGs were identified by comparing pairs of samples in the two sampling points (J1-J2, J1-J3, J1-J4, J1-J5, J1-J6, JCK-J4, X1-X2, X1-X3, X1-X4, X1-X5, X1-X6, XCK-X4) by using the DESeq R package. In addition, the false discovery rate $(\mathrm{FDR})<0.01 \& \mid \log 2$ (fold change) $\mid \geq 2$ was set as the threshold for significantly differential expression. $p$-values of the FDR were controlled using Benjaminie and Hodgberger's method; genes with a $p$-value $<0.01$ were considered differentially expressed.

\subsection{Self-Organizing Feature MapAnalysis}

A self-organizing feature map ( $\mathrm{SOM})$ is a data matrix and visualization method based on neural networks [23]. For the treatment methods of this experiment, SOM was used to design a formula containing the condition factor, time factor, and interaction between these factors. 'JWW' and ' $\mathrm{XBJ}$ ' DEGs were processed, normalized, and used in SOM machine learning for centralizing and clustering. Through this analysis, 21,035 DEGs were identified between 'JWW' and 'XBJ'. 21305 DEGs (Table S1) was normalized by using $\mathrm{R}$ language _scale, which is beneficial for the next step of SOM cluster analysis. The SOM approach was used to find a set of central points and map each object in the dataset to the corresponding central point under the most similar principle [24].

In neural network terminology, each neuron corresponds to a central point. The parameters for the SOM analysis were set as follows: som_model<-som (data_train_matrix, grid $=$ somgrid $(x d i m=30$, ydim $=30$, topo $=$ "hexagonal" $)$, rlen $=100$, alpha $=\mathrm{c}(0.05,0.01)$, keep.data $=$ TRUE). The SOMgrid defines the size of the network as $30 \times 30$ with six sides arranged between the squares. A matrix of the gene-to-sample expression values was used as input data. Genes with similar expression values were 
self-organized into the hexagon vicinity. The resulting map was visualized to show time-specific gene expression, in which a center point simplicity set defined multiple clusters that were grouped into the same cluster as the object closest to the center point. The expression model clustering was divided into each of the 20 'JWW' and 'XBJ' clusters.

\subsection{Gene Annotations andqRT-PCR}

Gene ontology (GO) enrichment analysis was conducted on the DEGs using GOseq R software [25]. KOBAS software was used to perform DEG enrichment statistics in the KEGG pathway [26]. Brassica Database v3.0 was used as reference lists [19]. Twelve DEGs were randomly selected to confirm the transcriptome data by qRT-PCR analysis. Gene-specific primers were designed using Primer v5.0 (Table S2). The normalized $e E F 1 B \alpha 2$ gene(BraA10g020830.3C.gene) was used as an internal control of gene expression [27]. The quality-assured RNA was used as template, with the synthesis of cDNA processed by a Takara PrimeScript RT reagent Kit (Takara, Nojihigashi, Kusatsu, Japan). The Bio-Rad CFX96 RT-PCR Detection system (Bio-Rad, Hercules, CA, USA) and SYBR Green II PCR Master mix (Takara) were used for the qRT-PCR reactions. The gene expression data were analyzed using the $2^{-\Delta \Delta C t}$ method [28]. SPSS v19.0 (SPSS, Chicago, IL, USA) was used to conduct the one-way analysis of variance (ANOVA) with Duncan's multiple range test using a significance threshold of $p<0.05$. The results were visualized using SigmaPlot v10.0 (Systat Software Inc., San Jose, CA, USA).

\section{Results}

\subsection{Confirmation of Investigation Periods}

From the beginning of vernalization to the end of vernalization, the number of leaves of 'JWW' is intuitively greater than that of ' $\mathrm{XBJ}$ ', which may be related to the degree of vernalization resistance (Figure 1A). At the same time, according to its own bolting characteristics, it can be seen that at least 25 days of vernalization can promote faster flowering of 'JWW', whereas ' $\mathrm{XBJ}$ ' only needs 10 days of vernalization to promote flowering (Figure 1B,C). For the comprehensive analysis of the differences in flowering times between the two B. rapa accessions, 14 different DAT [J1 (0 DAT), J2 (25 DAT), J3 (30 DAT), J4 (35 DAT), J5 (45 DAT), J6 (50 DAT), JCK (35 DAT at $\left.25^{\circ} \mathrm{C}\right), \mathrm{X} 1$ (0 DAT), X2 (10 DAT), X3 (15 DAT), X4 (25 DAT), X5 (40 DAT), X6 (50 DAT), XCK (25 DAT at $\left.25^{\circ} \mathrm{C}\right), \mathrm{J}$ for 'JWW', $\mathrm{X}$ for ' $\mathrm{XBJ}$ '] were selected for RNA sequencing (total of 42 samples). In 'JWW', RNA sequencing was selected based on the following criteria: (1) underwent at least 25 DAT before flowering; (2) 35 DAT promoted 'JWW' flowering relatively quickly; (3) according to the difference in flowering times near 35 DAT, 30 and 45 DAT, these times were selected as representatives; (4) 50 DAT was the maximum treatment time; (5) 0 and $35 \mathrm{DAT}$ at $25^{\circ} \mathrm{C}$ were used as the controls. In ' $\mathrm{XBJ}$ ', the criteria for RNA sequencing were as follows: (1) underwent at least 10 DAT before flowering; (2) 25 DAT promoted 'XBJ' flowering relatively quickly; (3) according to the difference in flowering times near 25 DAT, 15 and 40 DAT, these times were selected as representatives; (4) 50 DAT was the maximum treatment time; (5) 0 and 25 DAT at $25^{\circ} \mathrm{C}$ were used as the controls. 

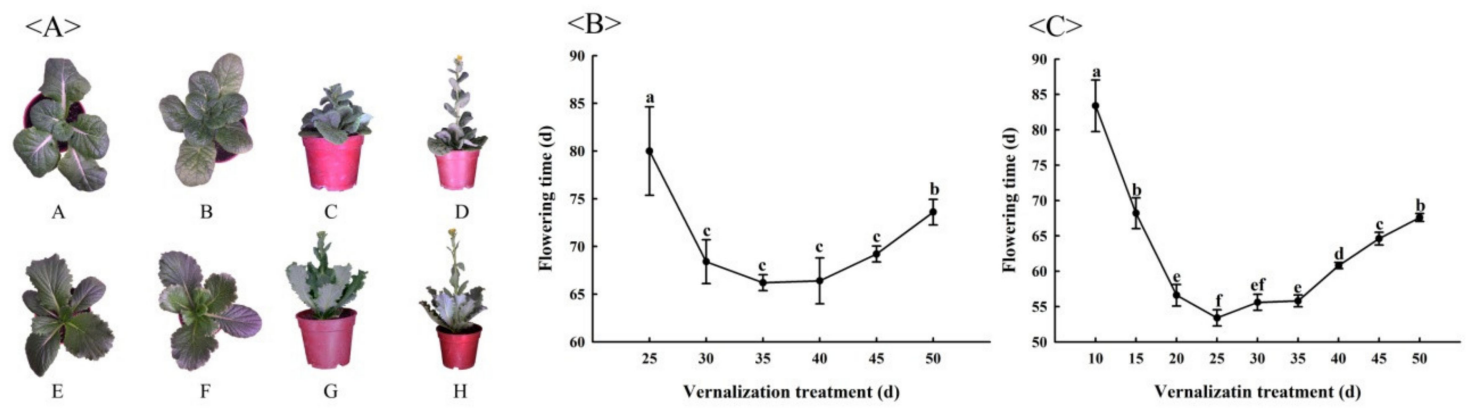

Figure 1. (A) The appearance of 'Jin Wawa' (JWW) (A-D) and 'Xiao Baojian' (XBJ) (E-H). (A), (E) Before vernalization (0 DAT); (B), (F) during vernalization (25 DAT); (C), (G) bolting; (D), (H) flowering. (B) The number of flowering time promoted by $4{ }^{\circ} \mathrm{C}$ vernalization treatment between 'JWW' and (C) 'XBJ'. Values represent the means of five replicates \pm standard error (SE) in the different vernalization periods. Values with the same letter are not significantly different at the $p<0.05$ level.

\section{2. $D E G$ Analysis of 'JWW' and ' $X B J^{\prime}$}

The two B. rapa accessions were analyzed across other periods using 0 DAT as the control for each period. Illumina RNA-Seq analysis of the 42 samples produced 1,949,168,044 total reads and $291.28 \mathrm{~Gb}$ clean data. The Q30 was $\geq 93.51 \%$ and the average GC content was $47.36 \%$ (Table S3A). After processing the reads, $974,584,022$ clean reads were obtained and sequence aligned with the designated B. rapa reference genome with an alignment efficiency ranging from $84.62 \%$ to $93.33 \%$. The unique map ratio ranged from $77.98 \%$ to $90.88 \%$, and the multiple map ratio ranged from $2.07 \%$ to $6.65 \%$ (Table S3B). Comparison with the B. rapa reference genome, 44,799 known genes and 2,280 new genes were identified.

Multiple groups were differentially analyzed using 0 DAT (J1 and X1) as the controls to identify vernalization-related DEGs (Figure 2). In 'JWW', 2245 common DEGs were continuously differentially expressed (822 upregulated; 1,403 downregulated) compared to J1. Fast flowering promoted at 35 DAT in 'JWW' uncovered 639 DEGs (392 upregulated; 305 downregulated). In 'XBJ', a total of 3278 DEGs (1267 upregulated; 2007 downregulated) were continuously expressed at 10, 15, 25, 40, and 50 DAT. A total of 486 DEGs (302 upregulated; 207 downregulated) were identified at 25 DAT, which promoted fast flowering in ' $\mathrm{XBJ}$ '. Clearly, ' $\mathrm{XBJ}$ ' possessed more continuously expressed genes during vernalization than 'JWW'. 

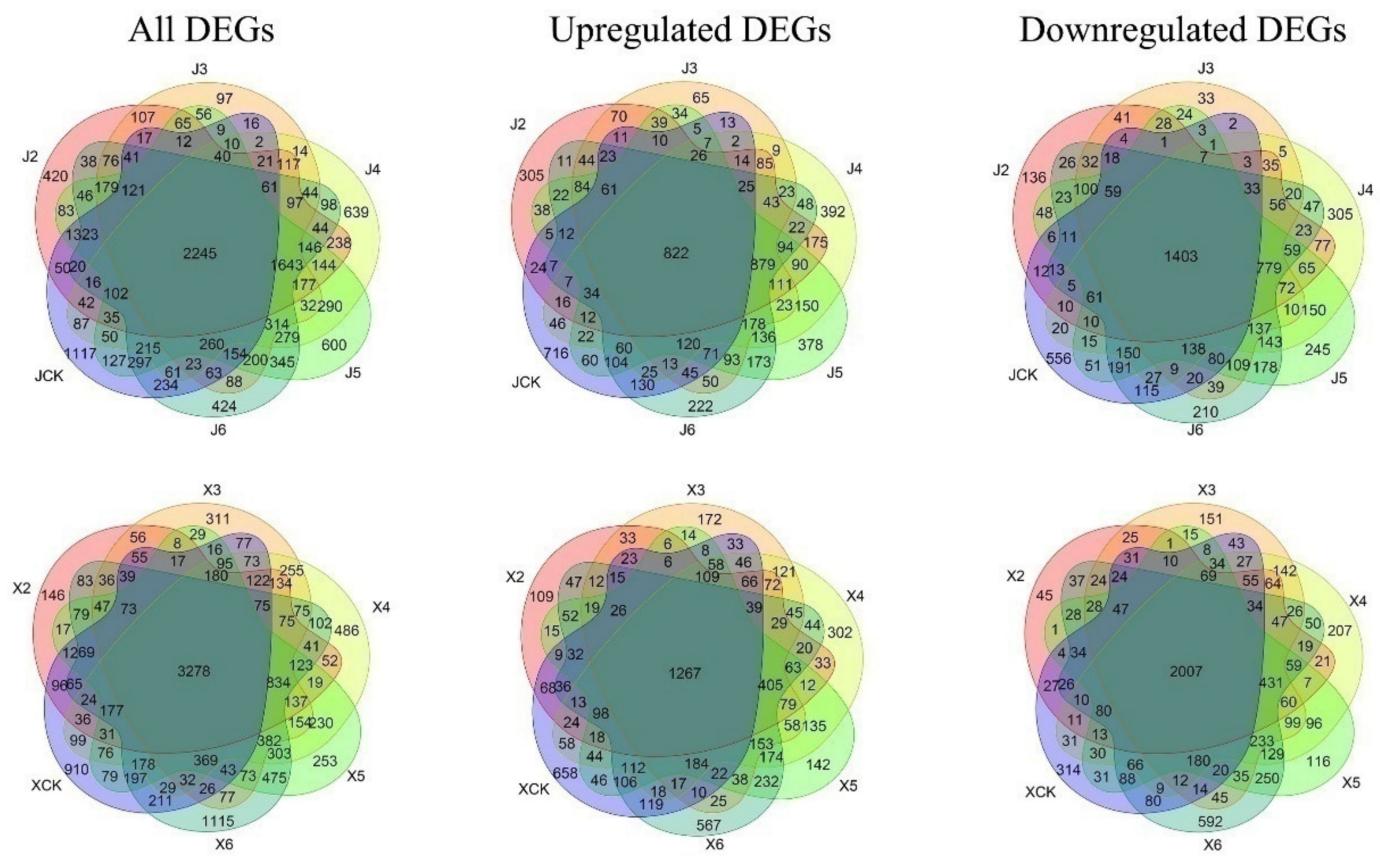

Figure 2. Venn diagrams of differentially expressed genes (DEGs) in the two B. rapa accessions during different vernalization periods. J2, J3, J4, J5, J6, and JCK represent the number of DEGs obtained by comparing 'JinWawa' 0 days after treatment (DAT) with 25, 30, 35, 40, and 50 DAT, and 35 DAT $25{ }^{\circ} \mathrm{C}$, respectively. $\mathrm{X} 2, \mathrm{X} 3, \mathrm{X} 4, \mathrm{X} 5, \mathrm{X} 6$, and $\mathrm{XCK}$ represent the number of DEGs obtained by comparing 'Xiao Baojian' 0 DAT with 10, 15, 25, 40, and 50 DAT and 25 DAT $25^{\circ} \mathrm{C}$, respectively. False discovery rate $(\mathrm{FDR})<0.01$ and $|\log 2 \mathrm{FC}|>1$ were used as cut-off criteria for significance.

To determine the functional distribution of unigenes, GO annotation was used to classify the DEGs generated by 'JWW' and ' $\mathrm{XBJ}$ ' during vernalization (Figure 3). The two B. rapa accessions exhibited a high degree of similarity in terms of unigene distribution. In the biological process category, unigenes were concentrated in the "metabolic process", "cellular process", and "single-organism process". In the cell component category, most unigenes were related to "cell", "organelle", and "cell part". In the molecular function category, unigenes were highly related to "catalytic activity" and "binding". These highly enriched GO terms indicated the basic regulatory and metabolic functions of the two B. rapa accessions during vernalization. For the metabolic processes in the biological category, we selected the vernalization periods common to 'JWW' and ' $\mathrm{XBJ}$ ' for comparison, which are 0 DAT (J1 and X1), 25 DAT (J2 and X4), and 50 DAT (J6 and X6). Compared to 0 DAT, we found that the change in 25 DAT and 50 DAT expression was larger in ' $\mathrm{XBJ}$ ' than in 'JWW'. Similarly, the catalytic activity-related genes in molecular functions are more pronounced in ' $\mathrm{XBJ}$ '. J2 and J6 are enriched in this term by 2723 and 3303 DEGs, respectively, compared with J1.X4 and X6 are enriched in this term with 3461 and 3630 DEGs, respectively, compared with X1. Overall, in these three GO analysis categories, the expression of ' $\mathrm{XBJ}$ ' in many terms has changed more than 'JWW'. 
A
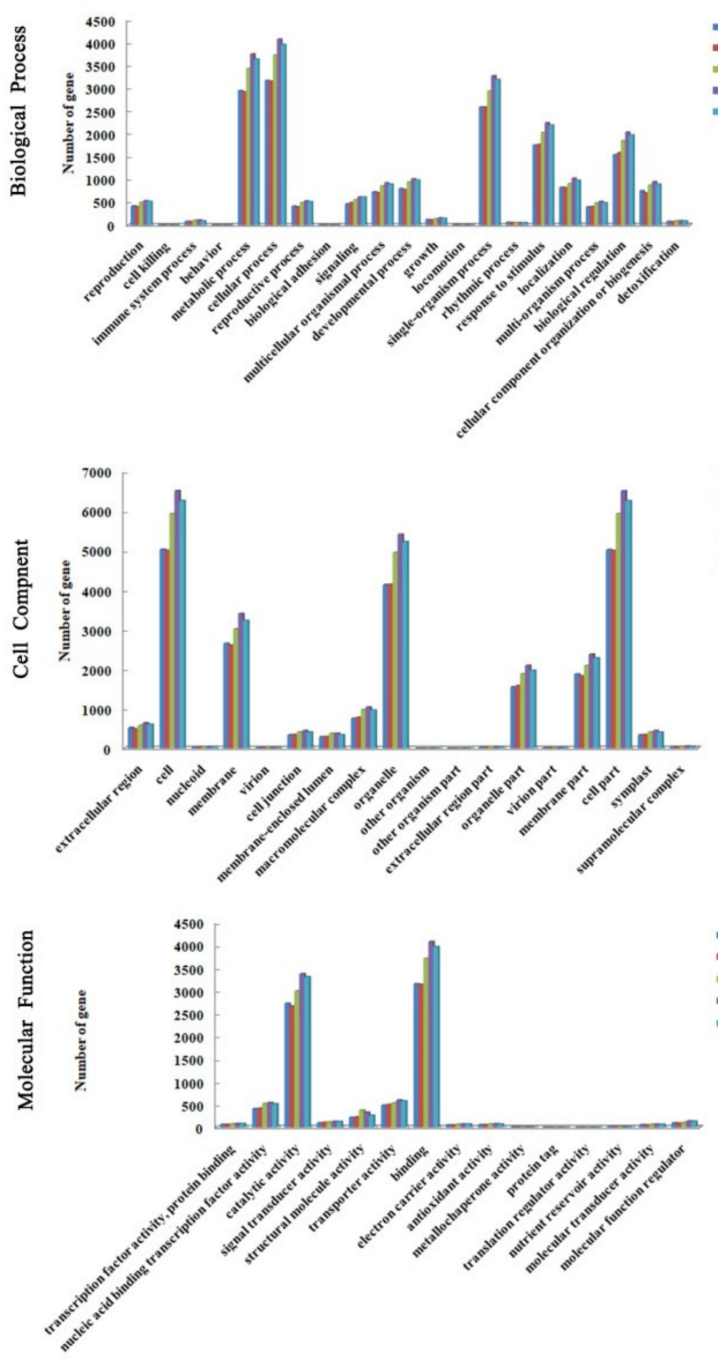

B
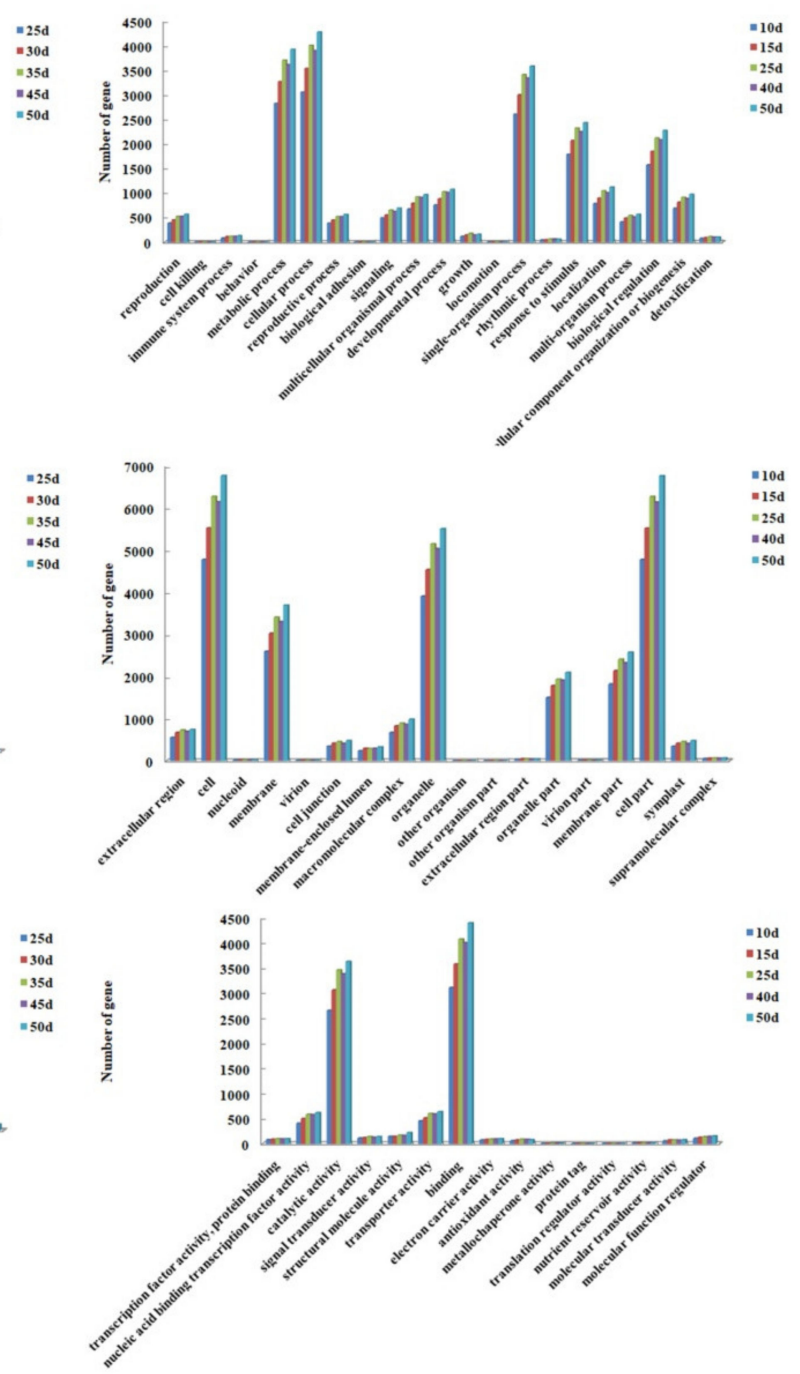

Figure 3. Gene ontology (GO) classification of the DEGs in 'JWW' (A) and 'XBJ' (B).

\subsection{Analyses of Dynamic Gene Expression Patterns during Vernalization}

To determine the main transcriptional dynamics related to vernalization of the two B. rapa accessions, SOM was used to identify the dynamic changes of the 21,035 DEGs in different vernalization periods, as well as a cluster analysis, which was performed on multiple groups of expression patterns. The expression of normalized DEGs in each period is represented by different colors of each hexagon in the square; the red to blue gradient indicated upregulation and downregulation, respectively (Figure 4A,C). Each DEG that exhibited a similar expression level was clustered into agrid and grids with the same color from each cluster were grouped together (Figure 4B,D). The characteristic values of the gene expression levels of each grid were extracted; 20 clusters were defined according to the number of grids (Figures S1 and S2). Then, GO classification analysis was performed on each cluster to analyze the regulated DEGs of the two B. rapa accessions during vernalization in the three GO categories (Tables S4 and S5). Clusters that were notably regulated during vernalization were selected to analyze the differential expression patterns of the two B. rapa accessions. 


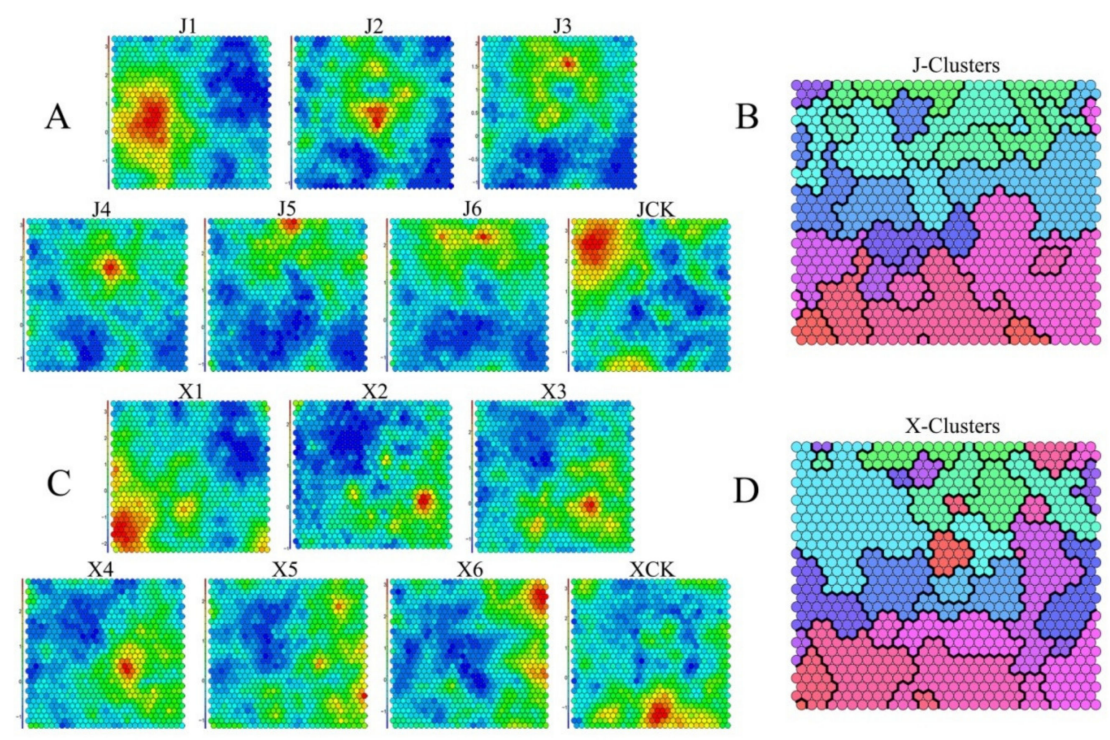

Figure 4. The temporal expression patterns of 21,035 DEGs in 'JWW' during vernalization (A); self-organizing feature map (SOM) clustering identified 20 clusters in 'JWW', in which different colors represent separate clusters (B); the temporal expression patterns of 21,035 DEGs in 'XBJ' during vernalization (C); SOM clustering identified 20 clusters in ' $\mathrm{XBJ}$ ', in which different colors represent separate clusters $(D)$. The red to blue gradient indicated upregulation and downregulation, respectively $(\mathrm{A}, \mathrm{C})$.

In clusters 7, 10, 11, 13, and 15 of 'JWW', comparing 0 DAT and 35 DAT $25^{\circ} \mathrm{C}$, DEGs exhibited a state of continuous induction during vernalization. These genes were upregulated starting at $25 \mathrm{DAT}$ and continued through 50 DAT. These continuously upregulated genes possessed biologically important functions and were used for studying the sensitivity of 'JWW' to vernalization. GO annotations were used for classifying the continuously increasing DEGs. Cellular components were enriched in genes related to extracellular space, lysosome, nucleolus, and endosome. In the biological processes category, DEGs were mainly enriched in regulation of gene expression and epigenetic. In the molecular functions category, these clusters had different DEGs that were enriched. Specifically, receptor activity-related genes were enriched in clusters 10,13, and 15. Clusters 7 and 11 were enriched in genes related to enzyme regulator activity. The genes related to binding, including oxygen and lipid binding, were enriched in each of the five clusters. In clusters 3, 12, and 19 of 'JWW', the DEGs remained stable and exhibited low expression levels at 25 DAT. Cluster 12 reached its lowest level at 50 DAT. The GO analysis revealed that genes related to extracellular space, nucleolus, and endosome in the cellular components category were enriched in these three clusters. Cluster 3 was enriched in genes involved in the regulation of gene expression and epigenetic in the biological processes category. The molecular functions category was enriched in activity, including receptor, nuclease, and enzyme regulator activities, and binding, including oxygen and lipid binding, genes in these three clusters.

In 'XBJ' at 10 DAT, clusters 7 and 10 were compared between 0 DAT and 25 DAT $25^{\circ} \mathrm{C}$, revealing that the genes were continuously upregulated. The GO analysis revealed that these genes were enriched in peroxisome and nucleoplasm in the cellular components category, cellular homeostasis in the biological processes category, and lipid binding in the molecular functions category; related genes were all enriched in these two clusters. Additionally, signal transducer activity and carbohydrate binding-related genes in the molecular functions category were enriched in cluster 10 of ' $\mathrm{XBJ}$ '. During the vernalization of ' $\mathrm{XBJ}$ ', clusters 1, 2, and 8 exhibited stable or low gene expression and were enriched in nucleoplasm of the cellular components category. During the vernalization of ' $\mathrm{XBJ}$ ', clusters 1,2, and 8 with stable or low gene expression were enriched in nucleoplasm of the cellular components category. Meanwhile, related genes of cluster 2 were enriched in golgi apparatus, and related genes of cluster 8 were enriched in nuclear envelope. Among the biological processes category, only cluster 8 , which 
contained low-expressing genes, was abundant in cellular homeostasis. Moreover, low-expressed genes were enriched only in cellular homeostasis. The molecular functions category was enriched in low-expressing genes related to signal transducer activity and lipid binding in clusters 1, 2, and 8 .

To further understand these continuously upregulated and downregulated genes in the enrichment pathways, these clusters were analyzed by a KEGG pathway analysis (Tables S6 and S7). In 'JWW', cluster 7 possessed continuously upregulated genes that were notably enriched in glycolysis/gluconeogenesis, starch and sucrose metabolism, and amino sugar and nucleotide sugar metabolism. In cluster 15, the DEGs of three aspects, including proteasome, plant hormone signal transduction, and glycolysis/gluconeogenesis, were notably expressed. Notably, during vernalization, these continuously upregulated clusters were considerably enriched in the plant hormone signal transduction and starch and sucrose metabolism pathways. Moreover, the plant hormone signal transduction and starch and sucrose metabolism pathways in clusters 3, 12, and 19 were continuously considerably downregulated. Analysis of the 'XBJ"s gene clusters that were continuously upregulated and downregulated during vernalization revealed that the plant hormone signal transduction and starch and sucrose metabolism pathways were also considerable. Therefore, a detailed analysis was performed on the related gene expression profiles of the plant hormone signal transduction and starch and sucrose metabolism pathways. Additionally, in order to fully explain the effect of vernalization on the two B. rapa accessions, the photoperiod, circadian clock, and vernalization pathways were incorporated in the analysis.

\subsection{DEGs of the Plant Hormone-Related Signal Transduction Pathway}

The plant hormone signal transduction pathways exhibited different DGEs levels during the two B. rapa accessions vernalization. To identify the hormone similarities and differences of the two different bolting B. rapa accessions, the expression levels of the hormone-related DEGs were further analyzed (Figure 5).

A total of 206 DEGs related to plant hormone signal transduction were identified in the two B. rapa accessions (Table S8). DEGs related to the plant hormone signaling pathway of 'JWW' and ' $\mathrm{XBJ}$ ' were enriched at various periods. During the transition from J1 (0 DAT) to J4 (35 DAT) in 'JWW', 44 and 40 of the 84 auxin-related DEGs were notably upregulated and downregulated, respectively. Among these 84 DEGs, 4 auxin transporter-like (AUX1), 9 auxin-responsive $(I A A), 3$ auxin response factor $(A R F), 2$ indole-3-acetic acid-amidosynthetase GH3 (GH3), and 6 auxin-responsive protein SAUR family genes $(S A U R s)$ were considerably upregulated $\left(\log 2\left(\mathrm{~J} 4_{\mathrm{FPKM}} / \mathrm{J} 1_{\mathrm{FPKM}}\right)>1\right)$. Moreover, 2 AUX1, 1 protein transport inhibitor response 1 (TIR1) (BraA07g025310.3C), 3 IAA, 1 ARF, 3 GH3, and 9 SAUR family genes were downregulated $\left(\log 2\left(\mathrm{~J} 4_{\mathrm{FPKM}} / \mathrm{J} 1_{\mathrm{FPKM}}\right)<-1\right)$. Interestingly, the upregulated and downregulation of these genes had the same change in J4 versus JCK. In ' $\mathrm{XBJ}$ ', 7 and 1 AUX1, 14 and $5 I A A, 4$ and $1 A R F, 4$ and 4 CH3, and 3 and 13 SAUR family genes were upregulated $\left(\log 2\left(X 4_{\mathrm{FPKM}} / \mathrm{X} 1_{\mathrm{FPKM}}\right)>1\right)$ and downregulated $\left(\log 2\left(\mathrm{X} 4_{\mathrm{FPKM}} / \mathrm{X} 1_{\mathrm{FPKM}}\right)<-1\right)$, respectively, in the transition from X1 (0 DAT) to X4 (25 DAT). These genes also had the same trend in X4 versus XCK. Seven DEGs were considerably expressed in the GA pathway. The two GA receptor GID1s were upregulated from J1 to J5 (0 to 45 DAT) in 'JWW', but clearly downregulated in J6 (50 DAT). In 'XBJ', the expression of GID1 genes varied throughout vernalization. Expression of the 2 GID2 genes in 'JWW' exhibited a fluctuating state of falling, rising, then falling and rising once more. Nevertheless, these levels rose after ' $\mathrm{XBJ}$ ' showed a decline. The three DELLA protein RG (DELLA) genes exhibited the same trend in both accessions. Two of the four ethylene response sensor (ETR) genes were upregulated and two were downregulated in the transition from J1 to J4 in the ethylene (ET) pathway of 'JWW'. In J4 versus JCK, all four ETRs were downregulated. In ' $\mathrm{XBJ}^{\prime}$, among the four ETRs, X4 versus X1 and X4 versus XCK only have one ETR downregulated and three upregulated. Notably, a mitogen-activated protein kinase 6 (MPK6) gene (BraA04g002220.3C.gene) was expressed in trace amounts in 'JWW', or was almost absent, but decreased considerably in the vernalization of ' $\mathrm{XBJ}$ '. ET-responsive transcription factor (ERF1/2) genes also behaved slightly differently in the B. rapa accessions. Compared to JCK, only 
one of the 17 abscisic acid (ABA) receptor PYL genes in the ABA pathway of 'JWW' was considerably upregulated in J4. In J4 versus J1, there were four PYL upregulated. Only one PYL (BraA10g000590.3C) remained the same upregulated level in both comparisons. However, compared to XCK, these PYL genes were upregulated and downregulated in ' $X \mathrm{BJ}$ ', respectively, which also occurred in $\mathrm{X} 4$ versus $\mathrm{X} 1$. Protein phosphatase 2C (PP2C) genes and serine/threonine-protein kinase (SnRK2) genes exhibited more consistent trends during vernalization. In 'JWW' compared to JCK at J4, most PP2C genes were considerably upregulated. Compared to $\mathrm{XCK}$ and $\mathrm{X} 4$, most $P P 2 \mathrm{C}$ genes were considerably downregulated in ' $\mathrm{XBJ}$ '. In the jasmonic acid (JA) pathway, 20 DEGs were identified. In the cytokinin (CK) pathway, 24 DEGs were identified, including 1 histidine kinase 4 X4 (AHK4), 3 histidine-containing phosphotransfer protein $(A H P)$, and 2 two-component response regulator ARR-B family $(B-A R R)$, and 13 two-component response regulator ARR-A family ( $A$-ARR) genes. In the salicylic acid (SA) pathway, the expression patterns of the eight transcription factor TGA genes were slightly different between the two accessions, which were more conspicuous in ' $\mathrm{XBJ}$ '.
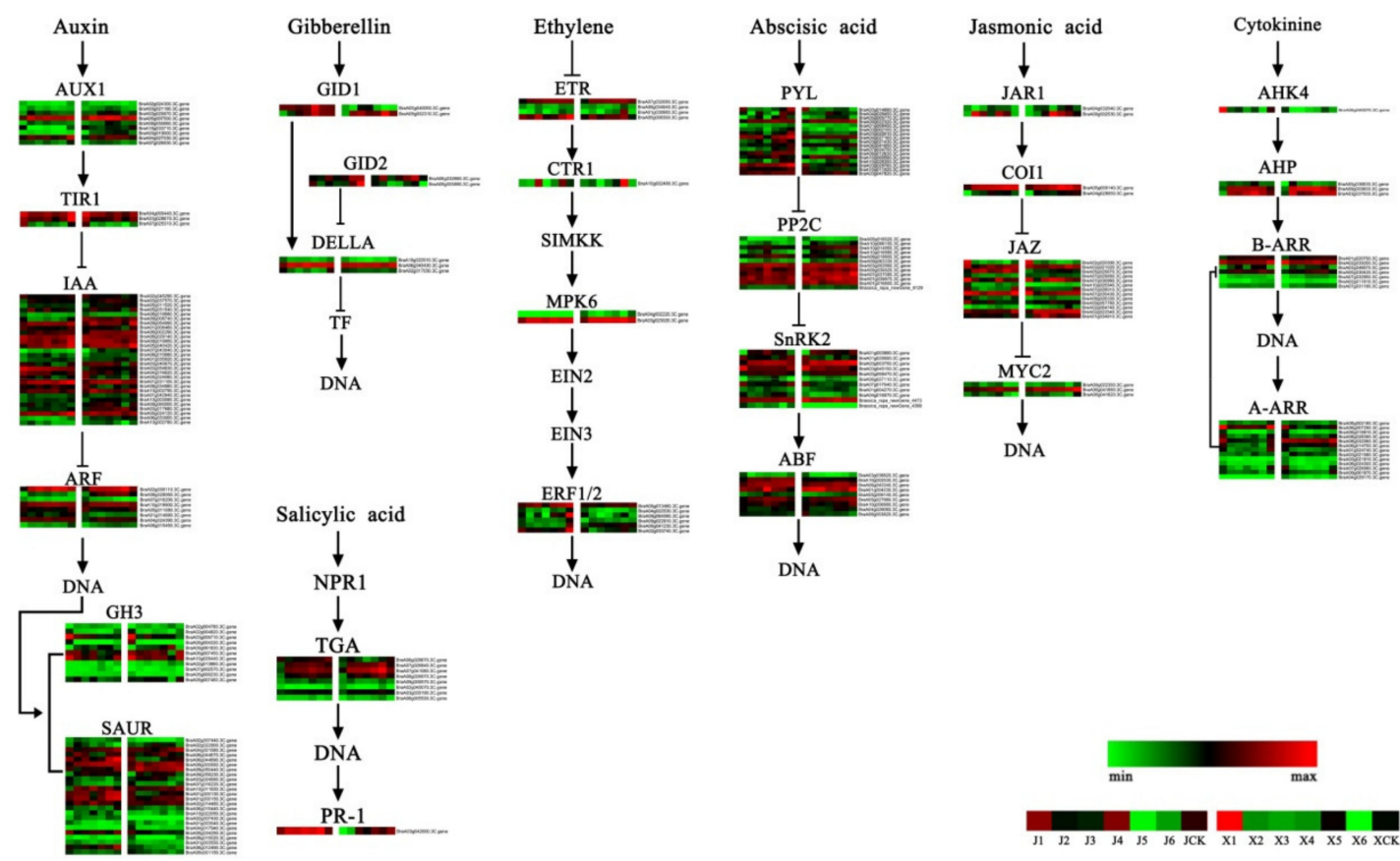

Figure 5. Heat map of plant hormone-related gene expression levels. Data were obtained using the $\log _{2}$ fragments per kilobase of exon per million fragments mapped (FPKM) of each gene. Red and green represent up- and downregulated genes, respectively. AUX1 = auxin transporter-like protein; TIR1 = protein transport inhibitor response 1; IAA = auxin-responsive protein IAA; ARF = auxin response factor; GH3 = indole-3-acetic acid-amido synthetase GH3; SAUR = auxin-responsive protein SAUR; GID1 = gibberellin receptor GID1; GID2 = gibberellin receptor GID2; DELLA = DELLA protein RG; NPR1 = nonexpresser of PR genes 1; TGA = transcription factor TGA; PR-1 = pathogenesis-related protein 1; ETR = ethylene receptor; CTR1 = serine/threonine-protein kinase CTR1; SIMKK = SIMK kinase; $\mathrm{MPK}=$ mitogen-activated protein kinase; EIN2 = ethylene-insensitive protein 2; EIN3 = ethylene-insensitive protein 3; ERF = ethylene-responsive transcription factor; PYL = abscisic acid receptor PYL; PP2C = protein phosphatase $2 \mathrm{C} ; \mathrm{SnRK} 2$ = serine/threonine-protein kinase SRK2; $\mathrm{ABF}=$ abscisic acid-insensitive; JAR1 = jasmonic acid-amido synthetase JAR1; COI1 = coronatine-insensitive protein 1; JAZ = protein TIFY; MYC2 = transcription factor MYC; AHK4 = histidine kinase 4; AHP = histidine-containing phosphotransfer protein; $\mathrm{A} / \mathrm{B}-\mathrm{ARR}=$ two-component response regulator ARR.

\subsection{Analysis of Starch and Sucrose Metabolism Pathways during Vernalization}

The enrichment pathways investigated by the KEGG pathway analysis included the starch and sucrose metabolism pathways, which were enriched in clusters 2, 7, and 10 of 'JWW', and in 
clusters 1, 2, and 10 of 'XBJ'. Subsequently, 134 DEGs in these pathways were further analyzed (Figure 6; Table S9). In the sucrose synthesis pathway, sucrose synthase (SUS) genes were notably expressed during vernalization in ' $\mathrm{JWW}^{\prime}$ and ' $\mathrm{XBJ}$ ', and its expression levels increased at first then decreased. However, 'JWW' did not express the newly discovered SUS gene (newGene_3912). Of the five sucrose-phosphate synthase (SPS) genes from J1 to J4, three were considerably upregulated $\left(\log 2\left(\mathrm{~J} 4_{\mathrm{FPKM}} / \mathrm{J} 1_{\mathrm{FPKM}}\right)>1\right)$, and one were considerably downregulated $\left(\log 2\left(\mathrm{~J} 4_{\mathrm{FPKM}} / \mathrm{J} 1_{\mathrm{FPKM}}\right)<-1\right)$ in JCK versus J4. In ' $\mathrm{XBJ}$ ' from $\mathrm{X} 1$ to $\mathrm{X} 4$, 4 were considerably upregulated $\left(\log 2\left(\mathrm{X} 4_{\mathrm{FPKM}} / \mathrm{X} 1_{\mathrm{FPKM}}\right)>1\right)$ and 1 was slight downregulated. The alpha-glucan phosphorylase (PYG) and 1,4-alpha-glucan-branching enzyme (GBE) genes in the starch synthesis pathway were notably different between the two $B$. rapa accessions. Expression of the three $P Y G$ and three GBE genes in each vernalization period of ' $X B J^{\prime}$ were expressed at higher levels than ' $J W W$ '. Vernalization promoted the upregulation of four maltose beta-amylase $(B A M)$ genes and downregulation of two genes. However, in the same period, five $B A M$ s were considerably higher than JCK in 'JWW' at J4. In X4, three BAM genes were expressed at higher levels in 'XBJ' than XCK. Alpha,alpha-trehalose-phosphate synthase (TPS) genes, which play important roles in sucrose and starch synthesis, were enriched in both accessions. Four TPSs in 'JWW' were considerably upregulated $\left(\log 2\left(\mathrm{~J} 4_{\mathrm{FPKM}} / \mathrm{J} 1_{\mathrm{FPKM}}\right)>1\right)$ and three were considerably downregulated $\left(\log 2\left(\mathrm{~J} 4_{\mathrm{FPKM}} / \mathrm{J} 1_{\mathrm{FPKM}}\right)<-1\right)$ from J1 to J4. It is worth noting that in J4 versus JCK, only two TPSs were considerably upregulated $\left(\log 2\left(\mathrm{~J} 4_{\mathrm{FPKM}} / \mathrm{J} 1_{\mathrm{FPKM}}\right)>1\right)$ and seven were considerably downregulated $\left(\log 2\left(\mathrm{~J} 4_{\mathrm{FPKM}} / \mathrm{J} 1_{\mathrm{FPKM}}\right)<-1\right)$. Unlike 'JWW', only seven TPSs were expressed, three of which were considerably upregulated $\left(\log 2\left(\mathrm{X} 4_{\mathrm{FPKM}} / \mathrm{X} 1_{\mathrm{FPKM}}\right)>1\right)$ in $\mathrm{X} 1$ to $\mathrm{X} 4$ of ' $\mathrm{XBJ}$ '. Only one TPS was considerably upregulated $\left(\log 2\left(\mathrm{X}_{\mathrm{FPKM}} / \mathrm{X} 1_{\mathrm{FPKM}}\right)>1\right)$ in $\mathrm{X} 4$ versus $\mathrm{XCK}$. Two of the three phosphoglucomutase $(\mathrm{pgm})$ genes increased and then decreased during vernalization. Two UTP-glucose-1-phosphate uridylyltransferase (UGP) genes, which play roles in the intermediate regulation of sucrose and starch synthesis, were expressed. Specifically, UGP1 was continuously downregulated and UGP2 was steadily upregulated as vernalization progressed, which was more evident in ' $\mathrm{XBJ}$ '. These results indicated that the starch and sucrose metabolism pathways play important roles in regulating the responses of $B$. rapa to vernalization. 


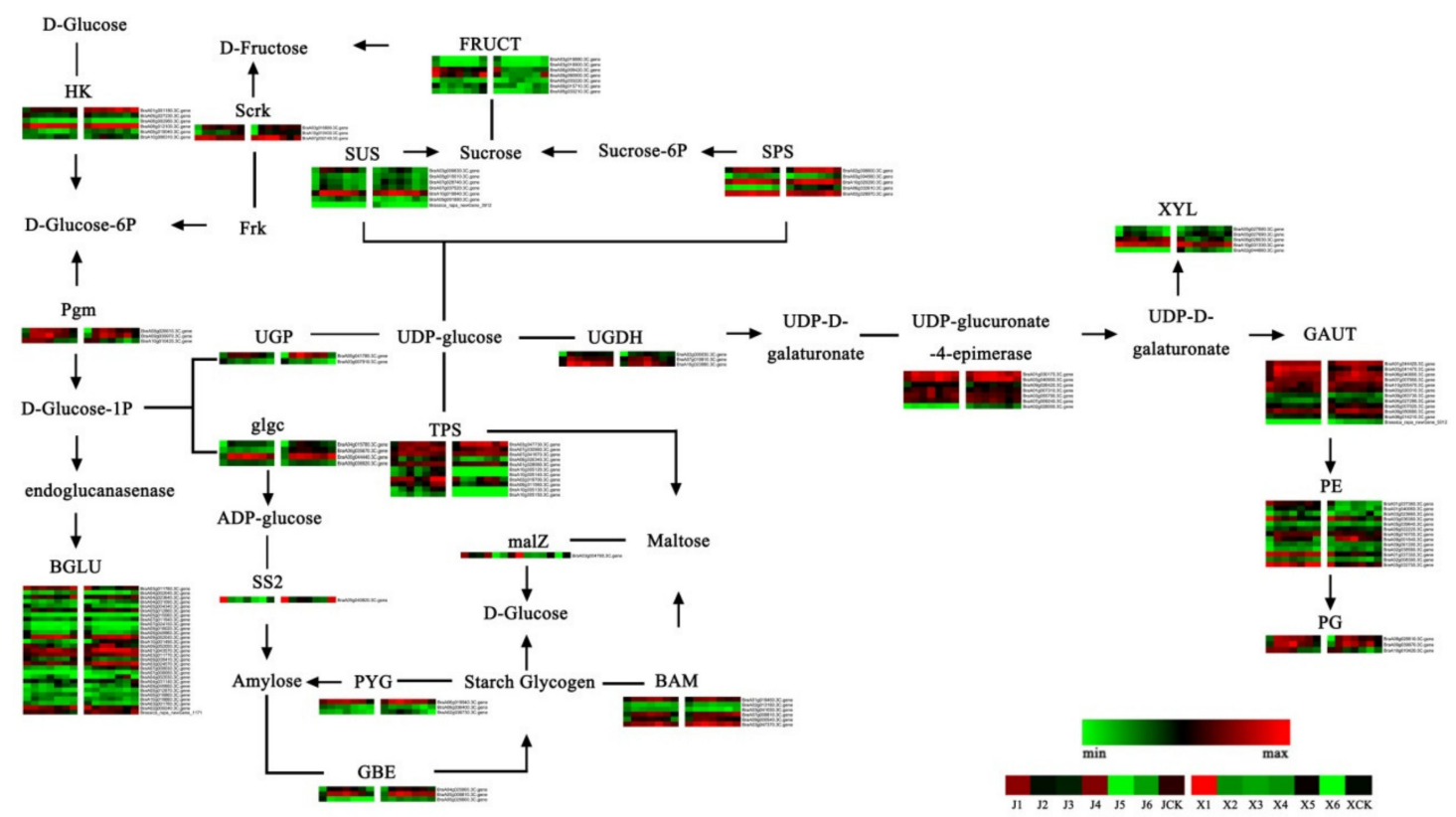

Figure 6. Heat map of the starch and sucrose-related gene expression levels. Data were obtained using the $\log _{2}$ FPKM of each gene. Red and green represent up- and downregulated genes, respectively. $\mathrm{HK}=$ histidine kinase; $\mathrm{BGLU}$ = beta-glucosidase; pgm = phosphoglucomutase; Scrk = fructokinase; SUS = sucrose synthase; UGP = UTP-glucose-1-phosphate uridylyltransferase; UDP = uridine diphosphate-glucose; UGDH = UDP-glucose 6-dehydrogenase; malZ = alpha-glucosidase; glgc = glucose-1-phosphate adenylyltransferase large subunit; SS2 = starch synthases 2; PYG = alpha-glucan phosphorylase; GBE = glucan-branching enzyme; Frk = fyn related Src family tyrosine kinase; $\mathrm{BAM}=$ maltose beta-amylase; $\mathrm{XYL}$ = beta-D-xylosidase; GAUT = beta-D-xylosidase; $\mathrm{PE}=$ pectinesterase/pectinesterase inhibitor; $\mathrm{PG}=$ phosphoglucomutase; $\mathrm{TPS}=$ trehalose-phosphate synthase; FRUCT = beta-fructofuranosidase; SPS = sucrose-phosphate synthase.

\subsection{DEGs of the Photoperiod and Circadian Clock Pathways}

The photoperiod and circadian clock pathway-related genes are often referred to as flowering-related genes. The two pathways of the red and blue light absorbing plants were compared and analyzed to the 23 flowering-related genes identified in this study (Figure 7; Table S10). In 'JWW' from $\mathrm{J} 1$ to $\mathrm{J} 4,12$ genes were considerably upregulated $\left(\log 2\left(\mathrm{~J} 4_{\mathrm{FPKM}} / \mathrm{J} 1_{\mathrm{FPKM}}\right)>1\right)$. In 'XBJ' from $\mathrm{X} 1$ to $\mathrm{X} 4$, only 7 genes were considerably upregulated $\left(\log 2\left(\mathrm{X} 4_{\mathrm{FPKM}} / \mathrm{X} 1_{\mathrm{FPKM}}\right)>1\right)$ and 6were considerably downregulated $\left(\log 2\left(\mathrm{X}_{\mathrm{FPKM}} / \mathrm{X}_{\mathrm{FPKM}}\right)<-1\right)$. Phytochrome $\mathrm{B}(\mathrm{PHY} B)$, two-component response regulator (APRR), and protein LHY (LHY) genes in the red-light pathway that positively regulate flowering locus $\mathrm{T}(F T)$ between the $B$. rapa accessions was slightly different during vernalization. It was worth noting that the two phytochrome B (PHYB) genes upregulated in J4 versus J1 were downregulated in J4 versus JCK. Ten APRRs were up- and downregulated during vernalization; the expression levels of APRRs in 'JWW' were generally higher than 'XBJ'. The expression of $L H Y S$, which play intermediate roles in the red-light pathway, were not notably different between the two accessions. The cryptochrome-2 (CRY), gigantea (GI), and cyclic dof factor (CDF) genes in the blue light pathway were negatively regulated. $C D F$ s were also regulated by red light-related genes. In the comparison between J4 and JCK, the GI and CDF genes in 'JWW' were upregulated during vernalization. Comparing $\mathrm{X} 4$ to $\mathrm{XCK}$, vernalization slightly upregulated the GIs of ' $\mathrm{XBJ}$ ', but downregulated CDFs. ' $\mathrm{XBJ}$ ' also upregulated flowering genes, including zinc finger protein constants $(\mathrm{CO})$ and FTs, during vernalization at higher levels than 'JWW'. These results indicated that the photoperiod and circadian clock pathways may play important roles in regulating $B$. rapa flowering during vernalization. 


\section{Red light}
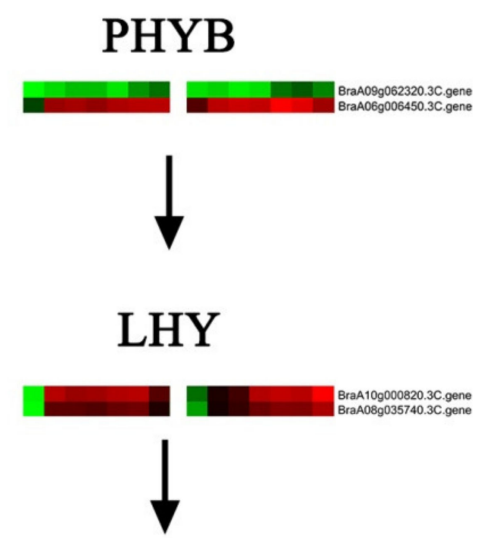

\section{Blue light}
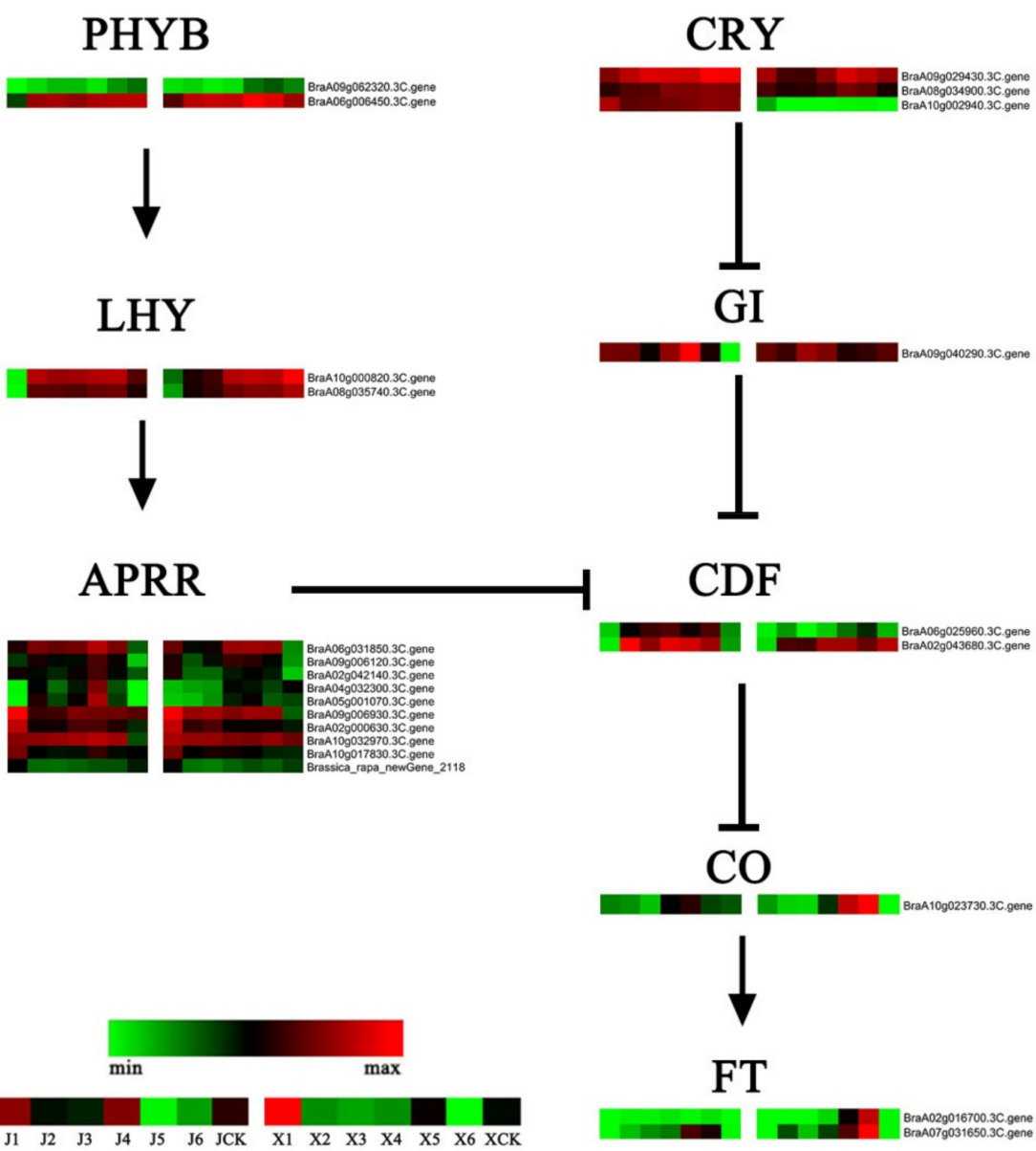

Figure 7. Heat map of the photoperiod and circadian clock gene expression levels. Data were obtained using the $\log _{2}$ FPKM of each gene. Red and green represent up- and downregulated genes, respectively. $\mathrm{PHYB}=$ phytochrome $\mathrm{B} ; \mathrm{LHY}=$ protein $\mathrm{LHY} ; \mathrm{APRR}=$ two-component response regulator; $\mathrm{CRY}=$ cryptochrome-2; $\mathrm{GI}=$ gigantea; $\mathrm{CDF}=$ cyclic dof factor; $\mathrm{CO}=$ constants; $\mathrm{FT}$ = floweing locus $\mathrm{T}$.

\subsection{Identification of VernalizationPathway DEGs}

Studies on the molecular mechanism of vernalization in dicotyledons have focused on the expression changes of FLC and its related regulatory genes. In this study, FLCS and their related vernalization regulating genes were identified (Figure 8; Table S11). VIN3, VRN2, and VRN1 genes were found to be negative regulators of $F L C$. Moreover, vernalization promoted the upregulation of VIN3s. The expression patterns of VRN1 between the two B. rapa accessions exhibited contrasting trends. Moreover, $V R N 2$ did not change much during vernalization. Vernalization reduced all four $F L C s$ and the reductions in ' $\mathrm{XBJ}$ ' were slightly greater than 'JWW'. FRI positively regulates FLC [10], but was not affected by vernalization. It can be observed that $F R I$ was not very different between $\mathrm{X} 4$ and $\mathrm{XCK}$, and there was not much difference in FRI expression between J4 and JCK. Although FRI expression in both accessions continuously increased as vernalization progressed, it could not be explained by the effects of vernalization. Downregulated $F L C s$ negatively affected the upregulation of $F T s$, which promoted the upregulation of the flowering factor, suppressor of constans overexpression 1 (SOC1). SOC1 in 'XBJ' was upregulated more than 'JWW'. 


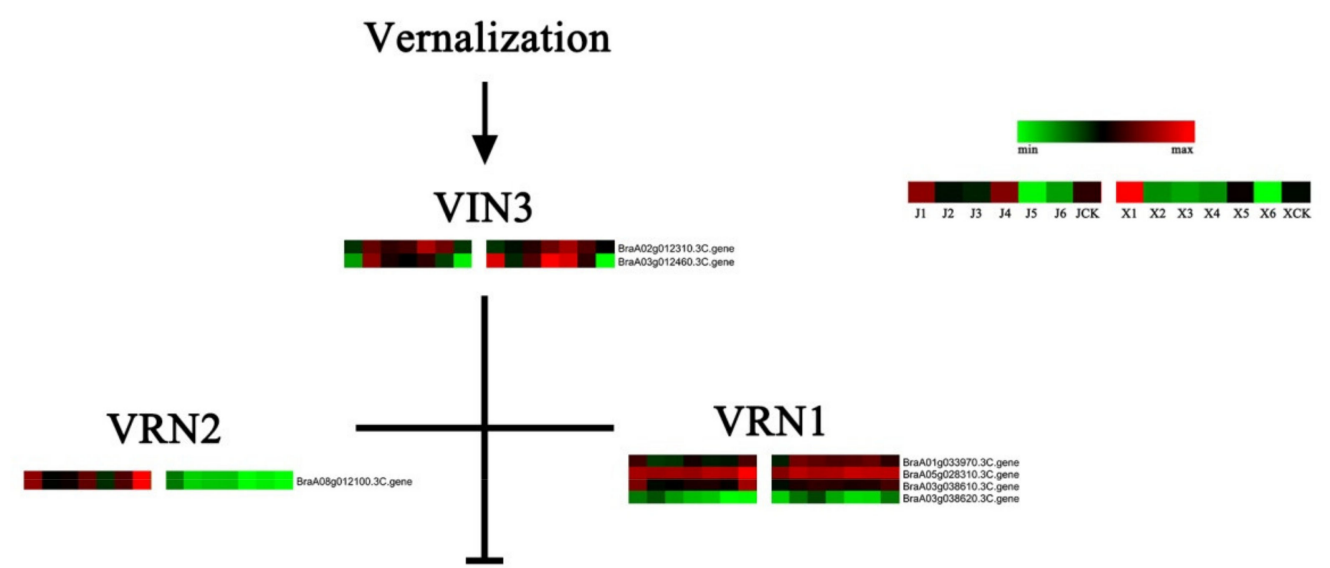

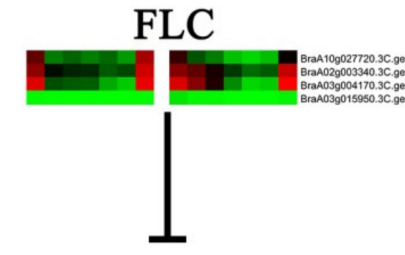

FT

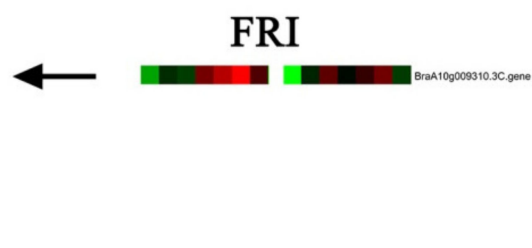

SOC1

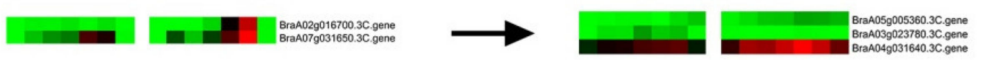

Figure 8. Map of vernalization-related gene expression levels. Data were obtained using the $\log _{2}$ FPKM of each gene. Red and green represent up- and downregulated genes, respectively. VIN3 = vernalization insensitive 3; VRN1 = B3 domain-containing transcription factor VRN1; VRN2 = B3 domain-containing transcription factor VRN2; FLC = flower locus C; FRI = frigida; SOC1 = suppressor of constans overexpression 1.

\section{8. qRT-PCRValidation of DEGs}

To verify the results of the RNA-Seq analysis, 12 DEGs were verified using qRT-PCR, including five plant hormone signal transduction (i.e., DELLA, PYL, JAR1, AHP, and TGA), three starch and sucrose metabolism (i.e., SPS, PYG, and BAM), two photoperiod and circadian clock (i.e., GI and $A P R R$ ), and two vernalization (i.e., VIN3 and FLC) genes (Figure 9). The 12 DEGs exhibited the same trends between the RNA-Seq and qRT-PCR results, indicating that the transcriptome analysis was accurate $\left(R^{2}=0.764\right.$; Figure S3). 

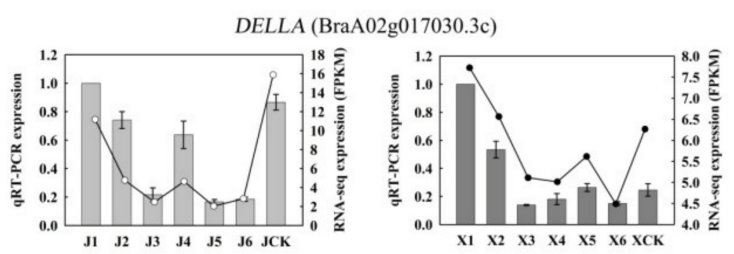

JAR1 (BraA09g002530.3c)
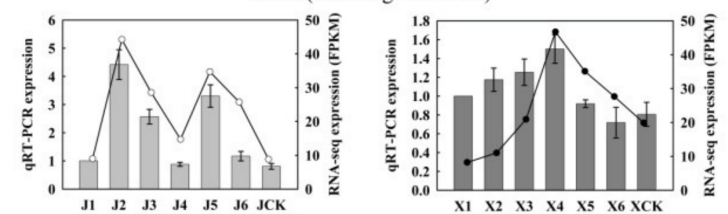

TGA (BraA07g026840.3C)
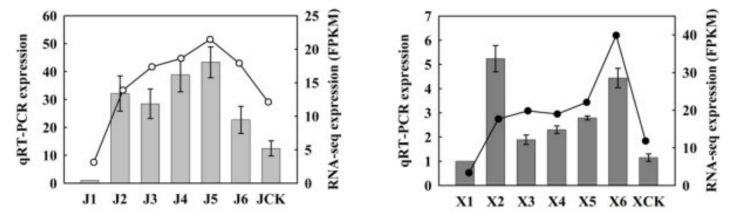

PYG (BraA06g019940.3c)

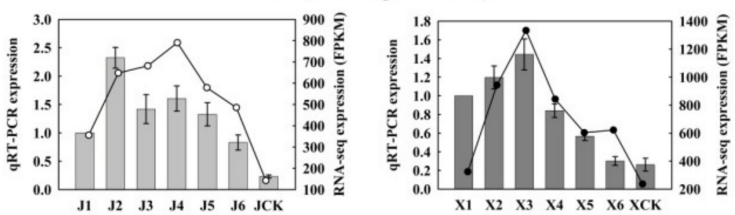

GI (BraA09g040290.3c)
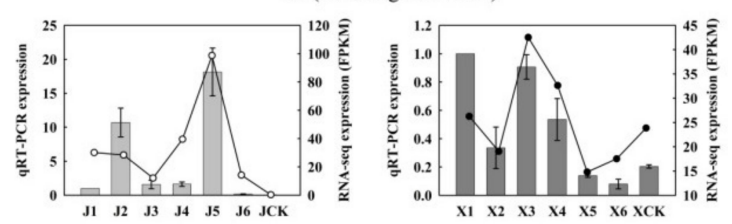

VIN3 (BraA02g012310.3c)
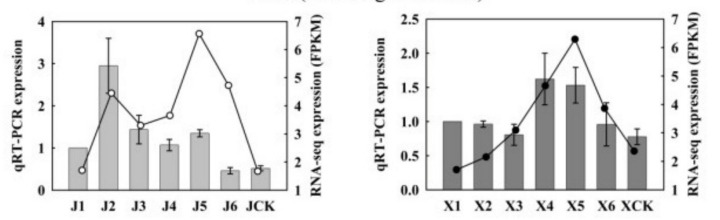
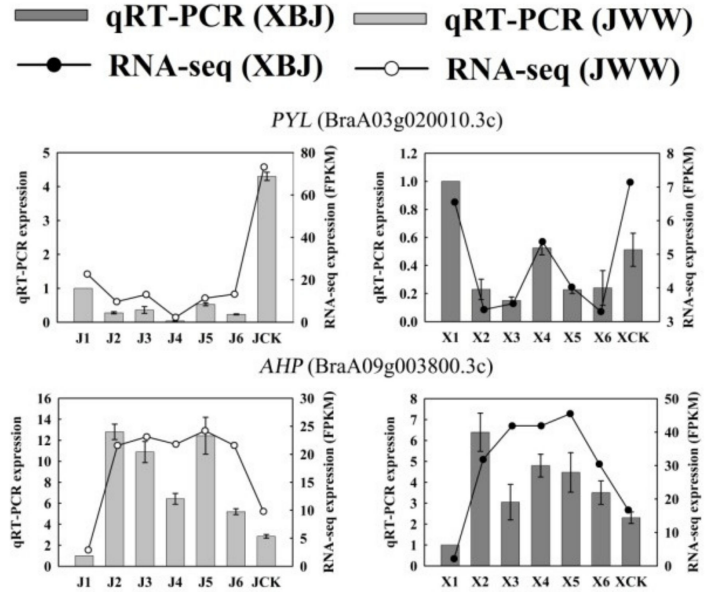

SPS (BraA02g008800.3C)

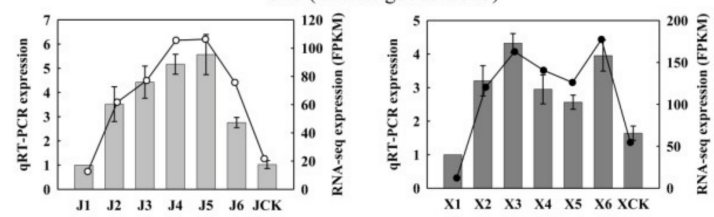

BAM (BraA03g047370.3c)

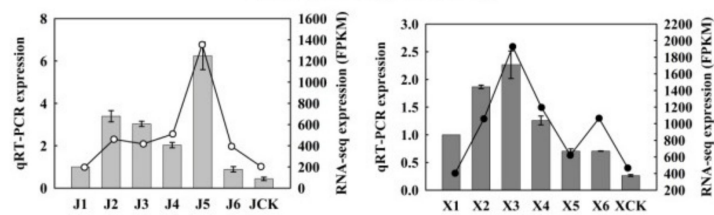

APRR (BraA06g031850.3c)

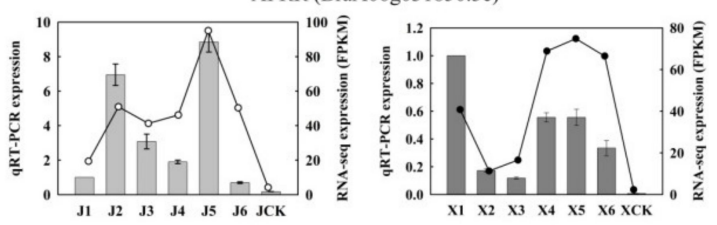

FLC (BraA03g004170.3C)

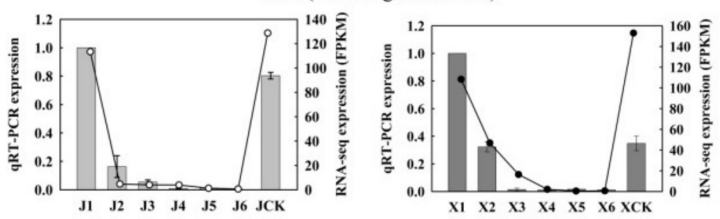

Figure 9. qRT-PCR was performed using 12 randomly selected DEGs. Bar and line graphs represent the qRT-PCR and RNA-Seq data, respectively. Data are presented as the mean \pm standard error (SE).

\section{Discussion}

Studies on transcriptome regulation based on phenotypes is a powerful research method $[29,30]$. Using this analytical method, DEGs and related regulatory pathways produced by Asiatic lily and tropical lotus during vernalization have been investigated [31,32]. With regard to B. rapa, the reference genome has been updated for several generations, and various regulatory pathways have been continuously studied $[33,34]$. However, only a few studies have been conducted on the vernalization control networks in B. rapa at different periods. Thus, in order to clarify the associated regulatory mechanisms, two bolting accessions were selected for transcriptome analysis in this study. A total of $974,584,022$ clean reads and $291.28 \mathrm{~Gb}$ clean data were obtained. After comparison with the B. rapa reference genome, 44,799 known genes and 2280 new genes were identified. A SOM analysis was performed and screened 21,036 DEGs in 'JWW' and 'XBJ' (Figure 4). GO and KEGG analyses were also performed on clusters that were continuously up- and downregulated during vernalization. DEGs 
that were enriched in the hormone signal transduction and sucrose metabolism pathways of these continuously up- and downregulated clusters were identified. Changes in the DEGs of these pathways during vernalization in the two B. rapa accessions were the primary focus of this study. Moreover, DEGs in the photoperiod and circadian clock, and vernalization pathways were analyzed.

\subsection{Changes in the Plant Hormone Signal Transduction Pathway during Vernalization}

The transition from vegetative to reproductive growth is a necessary process in plant flowering and reproduction. The shoot tip meristem (SAM) is an important part of the plant that helps complete the transition from vegetative to reproductive growth. During the vegetative growth stage, leaves formed around the SAM will generate lateral meristems. Then, a series of environmental influences and induced changes in endogenous signals will change the state of the SAM and induce flowering [35]. Plant hormones play a role in the flowering time syndrome of Arabidopsis [36]. However, in B. rapa, the regulation of hormones during vernalization remains unclear. Here, a layered heat map of the 7 plant hormone pathways of the two $B$. rapa accessions during vernalization was constructed to analyze this process in detail (Figure 5, Table S8).

Auxin functions in the initiation of floral primordia and flower development [37]. Aux/IAAs are primarily responsive to auxin genes. Aux/IAA stability and activity are regulated by auxin [38]. The interaction between Aux/IAA and TIR1 plays a role in regulating auxin signal transduction [39]. Mutations that occur in Aux/IAA and TIR1affect the interaction, resulting in low-auxin responses [40]. Similar to carbohydrates, auxin concentrations affect flower induction in different ways. Specifically, low concentrations promote flowering, while high concentrations delay flowering [36]. In Arabidopsis, the local accumulation of auxin releases $A R F 5$ due to $A u x / I A A$ inhibition, which subsequently promotes a rise in $C H 3$ levels, as well as high expression levels of $S A U R$ family genes, and is the leading cause of flowering on the lateral side of the SAM [41]. In this study, 'JWW' and ' $\mathrm{XBJ}^{\prime}$ ' upregulated $A R F S$, $\mathrm{CH} 3 \mathrm{~s}$, and $S A U R$ family genes with vernalization. These genes may be upregulated in the same way that auxin promotes flowering in Arabidopsis. This result indicates that, under the influence of vernalization, the auxin genes of the two B. rapa accessions may affect flower meristems, as well as their bolting and flowering abilities at different times.

GAs are a plant hormone involved in the floral transformation process of Arabidopsis and play a key role in controlling flowering time [2]. In Arabidopsis, GID1 is a positive regulator of GA signaling. The GID1-GA complex accelerates the degradation of the DELLA protein [42]. In this study, two GID1s were upregulated in the first four vernalization periods, but notably declined in the fifth period of 'JWW'. However, in 'XBJ', GID1 levels varied depending on the vernalization stage. The three $D E L L A s$ exhibited the same trend in both accessions. As vernalization continued, two DELLAs were downregulated and one was upregulated. These results indicated that the GA signaling pathway may play a role in the transition from the growth period during vernalization of these two accessions.

ET is a gas phytohormone that participates in plant stress responses and regulates a variety of developmental processes [43]. In Arabidopsis, the ET receptor possesses fivemembers and has been classified based on certain characteristics into ET receptor 1 (ETR1) and ET receptor 2 (ETR2) [44]. The serine/threonine-protein kinase CTR1 gene plays an important role in ET signal transduction [45]. There is a MPK module located between CTR1 and ethylene-insensitive protein 2 (EIN2) [46]. A previous study demonstrated that once MPK6 loses its function, the ET signaling pathway will return to normal, indicating that MPK6 may not participate in this pathway [47]. However, in the two B. rapa accessions of this study, 'XBJ' exhibited a large change in two MPK6s during vernalization compared to 'JWW'. Moreover, EIN2 is the positive regulator of the ET signaling pathway [48]. EIN3 is located downstream of EIN2. EIN2 and EIN3 work together and act on the ET-responsive transcription factor (ERF1/2) [49]. During vernalization, ERF1/2 genes were differentially expressed in the two $B$. rapa accessions of this study, which may explain the different conversion flowering periods.

ABA is a plant hormone that regulates plant development [50]. PYL family proteins are receptors of the ABA signaling pathway and inhibit PP2C in an ABA-dependent manner [51,52]. PP2Cs interact 
with SnRKs and inactivate them due to phosphorylation [53]. A previous study demonstrated that ABA played an inhibitory role in flowering time by modulating DELLA activities in the GA pathway [54]. A separate study found that ABA may play a role in hindering flowering and affect flowering by altering FLCS [55]. In this study, PYL genes were differentially expressed in both accessions and exhibited different trends. In the fourth period of vernalization, PYLs in 'JWW' were considerably downregulated compared to the control, while the PYLs in ' $X \mathrm{BJ}^{\prime}$ ' were upregulated and downregulated compared to the control. Moreover, vernalization continuously upregulated $P P 2 C$ genes, thereby blocking the generation of some SnRK2 genes; the difference was more noticeable in ' $\mathrm{XBJ}$ '. Therefore, the ABA signaling pathway may participate in regulating different growth period transformations in these two B. rapa accessions.

JA is a lipid plant hormone that plays multiple roles in plant growth and development [56]. CKs are a class of classical hormones that regulate both the division cycle and meristem homeostasis [57]. SAs are involved in plant defense functions and regulate leaf senescence [58]. In this study, the genes involved with these three plant hormones changed during vernalization. jasmonic acid-amido synthetase JAR1 (JAR1) is one of 19 closely related Arabidopsis genes that issimilar to the auxin-induced soybean GH3 gene [59]. Methyl JA (MeJA)-treated vernalization in insensitive spring wheat exhibited flowering and considerably downregulated TaVRN1 and TaFT1 genes, suggesting that MeJA may modulate vernalization and flowering time in wheat [60]. Previous studies have reported that CK promotes the formation of floral meristems and exogenously applied CK promotes Arabidopsis flowering [61,62]. The phytochrome signaling intersects with SA signaling [63], which correlates with flowering [64].

To summarize, different hormone biosynthesis and signaling pathways were differentially expressed during vernalization in the $B$. rapa accessions of this study. Clearly, this complex hormone signaling network accelerates the transition from vegetative to reproductive growth during vernalization in B. rapa.

\subsection{Gene Expression Changes in the Starch and Sucrose Metabolism Pathways}

The rapid propagation of plants is closely related to flowering time; carbohydrates are thought to play a crucial role in this process [65]. Sucrose and starch are the primary products of photosynthetic carbon assimilation. In lilies, sucrose treatment accelerates flowering [66]. Moreover, SUS is a key enzyme involved in the sucrose metabolism of plants [67], while SPS is a key enzyme that catalyzes the first step of the sucrose synthesis pathway [68]. 'XBJ' possessed a new SUS gene (newGene_3912), and the expression of SPSs in this accession were slightly higher than 'JWW' (Figure 6, Table S9).

Starch is the main carbohydrate stored, which allows them to meet their long-term carbon needs [69]. The main component of starch is synthesized by starch synthases (SS2), GBEs, and PYGs $[70,71]$. 'XBJ' highly expressed three PYGs and GBEs in each vernalization period. These differences indicated that the starch and sucrose metabolism pathways may play important roles in regulating $B$. rapa with different bolting times, but different roles in activating the SAM. Maltose is produced during the conversion of starch to sucrose in leaves under dark conditions [72]. BAM could potentially produce maltose as a key metabolite and osmolyte via starch hydrolysis [73]. Maltose is the primary source of carbon during cellular metabolism and growth at night [74]. Four BAMs were upregulated during vernalization in the two $B$. rapa accessions. However, when comparing the same vernalization period to the control, five $B A M$ s were upregulated in 'JWW' and three in ' $X \mathrm{BJ}$ '. These results indicated that maltose may provide the two $B$. rapa accession with sufficient energy required for normal growth during vernalization at night.

In Arabidopsis, TPS plays a critical role in controlling the transition from vegetative to reproductive growth $[75,76]$. TPS catalyzes the formation of trehalose-6-phosphate (T6P) from glucose-6-phosphate and uridine diphosphate-glucose (UDP-glu) and is a signaling molecule that relays information regarding carbohydrate availability to other signaling pathways [77,78]. The loss of TPS resulted in extremely delayed flowering in Arabidopsis. The TPS pathway also acts as a signal that coordinates 
the induction of flowering by regulating the expression of key floral integrators in the leaves and SAM [79]. In this study, the TPSs of 'XBJ' expressed during vernalization were less than 'JWW'. It may be that the late-bolting 'JWW' accession requires higher TPS expression in order to promote faster flowering. The upregulation of $p g m s$ promotes the production of polysaccharides, which has been demonstrated in mushrooms [80]. In this study, pgms increased at first and then decreased during vernalization. Therefore, it is speculated that $p g m s$ rose rapidly at the beginning of vernalization in order to provide sufficient energy for normal growth, and then it gradually decreased after the vernalization transformation period. In young and mature leaves, UGP is primarily involved in the sucrose biosynthesis pathway, the major form of transport of carbon in plants, which provides energy to plants [81]. In this study, two UGP genes exhibited opposing states during vernalization, in which, one was upregulated and the other was downregulated. The underlying mechanisms for these contrasting trends, however, require further investigation.

\subsection{Photoperiod and Circadian Clock Pathways}

The photoperiod and circadian clock pathways function as a whole, as plants coordinate their metabolism according to changes in photoperiods, which thereby induces flowering. Expression changes in the CO/FT mode is the core link between the photoperiod induction pathway [3]. The purpose of this study was to analyze the influence of vernalization on the related genes in this pathway under certain photoperiods (Figure 7, Table S10). CO is a typical circadian clock control gene that acts on the $F T$ promoter region and is the main positive regulator of FT [82]. The red-light pathway positively regulates $C O$ and $F T$, which were continuously regulated by $C D F s$ through PHYBs, LHYs, and APRRs. Among them, APRRs were considerably different between the two $B$. rapa accessions and may affect bolting. The two PHYBs upregulated in J4 versus J1 were downregulated in J4 versus JCK, suggesting that $P H Y B$ s may not be affected by vernalization in 'JWW'. CDFs are intermediate regulatory genes in this pathway that are regulated by both red and blue light. Specifically, vernalization downregulated the CDFs of ' $\mathrm{XBJ}$ ', but upregulated the CDFs of 'JWW'. Thus, CDF may be a gene that does not affect late-bolting B. rapa during vernalization.

\subsection{DEGs of the Vernalization Pathway}

Land plant flower development undergoes floral induction, floral primordia, and floral organ development phases. In the flowering induction stage, although there are no morphological changes in the SAM, a series of related genes centered around FT undergo various changes, forming a complex regulatory network to achieve the transition from vegetative to reproductive growth [83]. In $B$. rapa, vernalization is the most important process that induces flowering. Currently, studies on the underlying molecular mechanism of the vernalization pathway in A. thaliana mainly focus on changes in the expression levels of FLCs. Among them, BrFLC1 and BrFLC2 of B. rapa respond to the regulation of vernalization and affect flowering time [84]. In this study, three of the four FLCs decreased considerably during vernalization. The decrease in ' $\mathrm{XBJ}$ ' was larger than that in 'JWW', and it continually decreased in the first five vernalization periods (Figure 8, Table S11). VRN1, VRN2, and VIN3 are negative regulators of FLC, and previous studies demonstrated that VRN1 and VRN2 in Arabidopsis were not induced by vernalization and only play a role in the persistently low expression of FLC at the end of vernalization [12-14]; thus, the results of this study were inconsistent, as two B. rapa VRN1s exhibited opposing modes during vernalization. However, these differences require further investigation. Changes in VRN2 were consistent with previous results [13]. VIN3s were upregulated in the first five vernalization periods and downregulated in the last vernalization period. Therefore, it is speculated that B. rapa transitioned to reproductive growth just before the last vernalization period and, hence, the downregulation of VIN3s. 


\section{Conclusions}

To date, studies on the mechanisms related to vernalization of B. rapa have focused only on the comparison between the vernalized and unvernalized, and related research approaches have been relatively narrow. Vernalization promotes plant bolting and flowering as an important trait in productivity and reproduction; thus, it is necessary to clarify its effects on bolting and flowering in different vernalization periods. In this study, two B. rapa accessions with different bolting times were investigated over a series of vernalization periods. For the related flowering traits, the vernalization periods were selected for comparative transcriptome analysis to describe the effects of different vernalization periods on B. rapa. According to the results, the B. rapa accessions exhibited specific differences at each vernalization period, indicating that research on vernalization should be a process of vernalization, not a comparison between vernalized and unvernalized. In comparing the two accessions, key genes of seven hormone pathways of the plant hormone signal transduction pathway during vernalization were notably different, and the difference between late-bolting ' $\mathrm{XBJ}$ ' was greater than early-bolting 'JWW'. This difference was also confirmed in the vernalization pathway. In the starch and sucrose metabolism and photoperiod and circadian clock pathways, TPS, UGP, and CDF exhibited different expression patterns, but these differences require further analysis. Notably, VIN1, which does not play a role during vernalization in other dicotyledons, exhibited opposing expression patterns in this study, which may be a new avenue for research on vernalization in $B$. rapa in the future. The transcriptional regulation of B. rapa vernalization was summarized in this study (Figure 10), which provides a foundation for future investigations on the molecular mechanisms of $B$. rapa with different abstractions during vernalization.

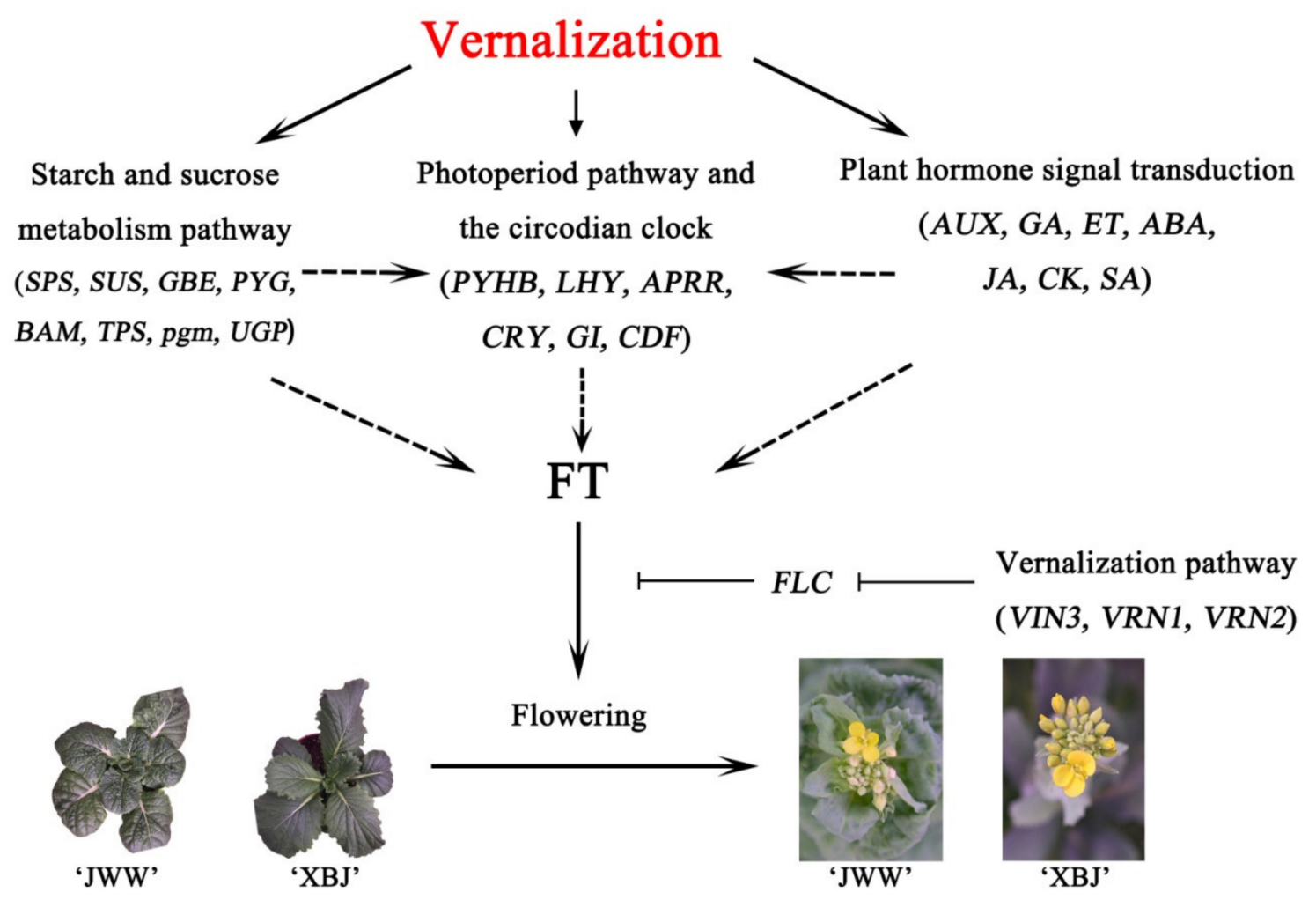

Figure 10. Summary of vernalization transcript regulation in B. rapa. Black arrows indicate promotion, and inverted T bars indicate repression. Dashed lines indicate that further research is needed. 
Supplementary Materials: The following are available online at http://www.mdpi.com/2073-4425/11/4/392/s1, Figure S1. Clusters expressions associated with 'JWW' vernalization periods. The x-axis represents vernalization periods, and the $y$-axis represents expression. Figure S2. Clusters expressions associated with 'XBJ' vernalization periods. The $\mathrm{x}$-axis represents vernalization periods, and the $\mathrm{y}$-axis represents expression. Figure S3. Linear relationships between qRT-PCR data and RNA-Seq data of related genes. Table S1. The 21035DEGs in both B. rapa accessions at different periods ( $\mathrm{J}$ for 'JWW'; $\mathrm{X}$ for ' $\mathrm{XBJ}$ '). Table S2. Specific primers for differential gene sequences for qRT-PCR. Table S3. (A) Statistics of Illumina sequencing data (J for 'JWW'; $X$ for ' $X \mathrm{BJ}$ '); (B) The sequencing and mapped results obtained from each sample ( $\mathrm{J}$ for ' $J W W^{\prime}$ '; $\mathrm{X}$ for ' $\mathrm{XBJ}$ '). Table S4. GO classification of DEGs in each clusters for the 'JWW'. Red indicates the number of terms genes that continuously increase; blue indicates the number of terms genes that continuously decrease. Table S5. GO classification of DEGs in each clusters for the ' $\mathrm{XBJ}$ '. Red indicates the number of terms genes that continuously increase; blue indicates the number of terms genes that continuously decrease. Table S6. Analysis of pathways involving up- and downregulated genes for clusters in 'JWW'. Table S7. Anlysis of pathways involving up- and downregulated genes for clusters in 'XBJ'.Table S8. Plant hormone related DEGs in two B. rapa accessions during the vernalization process. Table S9. Starch and sucrose related DEGs in two B. rapa accessions during the vernalization process. Table S10. Photoperiod and circadian clock related DEGs in two B. rapa accessions during the vernalization process. Table S11. Vernalization related DEGs in two $B$. rapa accessions during the vernalization process.

Author Contributions: Data curation: Y.D. and X.S.; Funding acquisition, S.Z. (Shujiang Zhang) and R.S.; Methodology, X.S., F.L. and H.Z.; Project administration, R.S.; Resources, G.L.; Software, L.Y., S.Z. (Shifan Zhang) and G.C.; Supervision, C.W. and R.S.; Validation, C.W.; Writing-original draft, Y.D. and S.Z. (Shujiang Zhang); Writing-review \& editing, S.Z. (Shujiang Zhang) and G.L. All authors have read and agreed to the published version of the manuscript.

Funding: This work was funded by China Agricultural Research System (CARS-25-A-01) and the National Key Research and Development Program of China (grant number 2017YFD0101802).

Acknowledgments: We thank LetPub (www.letpub.com) for its linguistic assistance during the preparation of this manuscript.

Conflicts of Interest: The authors declare that they have no competing interests.

\section{References}

1. Amasino, R. Seasonal and developmental timing of flowering. Plant J. 2010, 61, 1001-1013. [CrossRef] [PubMed]

2. Wilson, R.N.; Heckman, J.W.; Somerville, C.R. Gibberellin Is Required for Flowering in Arabidopsis thaliana under Short Days. Plant Physiol. 1992, 100, 403-408. [CrossRef] [PubMed]

3. Turck, F.; Fornara, F.; Coupland, G. Regulation and identity of florigen: FLOWERING LOCUS T moves center stage. Annu. Rev. Plant Biol. 2008, 59, 573-594. [CrossRef] [PubMed]

4. Jung, C.; Muller, A.E. Flowering time control and applications in plant breeding. Trends Plant Sci. 2009, 14, 563-573. [CrossRef]

5. He, Y. Chromatin regulation of flowering. Trends Plant Sci. 2012, 17, 556-562. [CrossRef]

6. Wang, J. Regulation of flowering time by the miR156-mediated age pathway. J. Exp. Bot. J. 2014, 65, 4723-4730. [CrossRef]

7. Srikanth, A.; Schmid, M. Regulation of flowering time: All roads lead to Rome. Cell. Mol. Life Sci. 2011, 68, 2013-2037. [CrossRef]

8. Han, T.; Wu, C.; Tong, Z.; Mentreddy, R.S.; Tan, K.; Gai, J. Postflowering photoperiod regulates vegetative growth and reproductive development of soybean. Environ. Exp. Bot. 2006, 55, 120-129. [CrossRef]

9. Xu, F.; Rong, X.; Huang, X.; Cheng, S. Recent advances of flowering locus T gene in higher plants. Int. J. Mol. Sci. 2012, 13, 3773-3781. [CrossRef]

10. Johanson, U.; West, J.; Lister, C.; Michaels, S.D.; Amasino, R.M.; Dean, C. Molecular Analysis of FRIGIDA, a Major Determinant of Natural Variation in Arabidopsis Flowering Time. Science 2000, 290, 344-347. [CrossRef]

11. Gendall, A.R.; Levy, Y.Y.; Wilson, A.; Dean, C. The VERNALIZATION 2 Gene Mediates the Epigenetic Regulation of Vernalization in Arabidopsis. Cell 2001, 107, 525-535. [CrossRef]

12. Levy, Y.Y.; Mesnage, S.; Mylne, J.S.; Gendall, A.R.; Dean, C. Multiple Roles of Arabidopsis VRN1 in Vernalization and Flowering Time Control. Science 2002, 297, 243-246. [CrossRef] [PubMed]

13. Yan, L.; Loukoianov, A.; Blechl, A.; Tranquilli, G.; Ramakrishna, W.; SanMiguel, P.; Bennetzen, J.L.; Echenique, V.; Dubcovsky, J. The Wheat VRN2 Gene is a Flowering Represser Down-Regulated by Vernalization. Science 2004, 303, 1640-1644. [CrossRef] [PubMed] 
14. Kim, D.H.; Zografos, B.R.; Sung, S. Mechanisms underlying vernalization-mediated VIN3 induction in Arabidopsis. Plant Signal. Behav. 2014, 5, 1457-1459. [CrossRef] [PubMed]

15. Shitsukawa, N.; Ikari, C.; Shimada, S.; Kitagawa, S.; Sakamoto, K.; Saito, H.; Ryuto, H.; Fukunishi, N.; Abe, T.; Takumi, S.; et al. The einkorn wheat (Triticum monococcum) mutant, maintained vegetative phase, is caused by a deletion in the VRN1 gene. Genes Genet. Syst. 2007, 82, 167-170. [CrossRef] [PubMed]

16. Kiss, T.; Dixon, L.E.; Soltész, A.; Bányai, J.; Mayer, M.; Balla, K.; Allard, V.; Galiba, G.; Slafer, G.A.; Griffiths, S.; et al. Effects of ambient temperature in association with photoperiod on phenology and on the expressions of major plant developmental genes in wheat (Triticumaestivum L.). Plant Cell Environ. 2017, 40, 1629-1642. [CrossRef]

17. Yan, L.; Fu, D.; Li, C.; Blechl, A.E.; Tranquilli, G.; Bonafede, M.; Sanchez, A.; Valarik, M.; Yasuda, S.; Dubcovsky, J. The wheat and barley vernalization gene VRN3 is an orthologue of FT. Proc. Natl. Acad. Sci. USA 2006, 103, 19581-19586. [CrossRef]

18. Li, J.; Zhao, X.; Nishimura, Y.; Fukumoto, Y. Correlation between bolting and physiological properties in Chinese cabbage (Brassica rapa L. pekinensis group). J. Jpn. Soc. Hortic. Sci. 2010, 79, 294-300. [CrossRef]

19. Zhang, L.; Cai, X.; Wu, J.; Liu, M.; Grob, S.; Cheng, F.; Liang, J.; Cai, C.; Liu, Z.; Liu, B. Improved Brassica rapa reference genome by single-molecule sequencing and chromosome conformation capture technologies. Hortic. Res. 2018, 5, 1-11. [CrossRef]

20. Trapnell, C.; Pachter, L.; Salzberg, S.L. TopHat: Discovering splice junctions with RNA-Seq. Bioinformatics 2009, 25, 1105-1111. [CrossRef]

21. Trapnell, C.; Roberts, A.; Goff, L.A.; Pertea, G.; Kim, D.; Kelley, D.R.; Pimentel, H.; Salzberg, S.L.; Rinn, J.L.; Pachter, L. Differential gene and transcript expression analysis of RNA-seq experiments with TopHat and Cufflinks. Nat. Protoc. 2012, 7, 562-578. [CrossRef] [PubMed]

22. Anders, S.; Huber, W. Differential expression analysis for sequence count data. Genome Biol. 2010, 11, 1-12. [CrossRef] [PubMed]

23. Gerber, T.; Willscher, E.; Loefflerwirth, H.; Hopp, L.; Schadendorf, D.; Schartl, M.; Anderegg, U.; Camp, G.; Treutlein, B.; Binder, H.; et al. Mapping heterogeneity in patient-derived melanoma cultures by single-cell RNA-seq. Oncotarget 2017, 8, 846-862. [CrossRef] [PubMed]

24. Chang, R.; Chu, C.; Wu, Y.; Chen, Y. Gene clustering by using query-based self-organizing maps. Expert Syst. Appl. 2010, 37, 6689-6694. [CrossRef]

25. Young, M.D.; Wakefield, M.J.; Smyth, G.K.; Oshlack, A. Gene ontology analysis for RNA-seq: Accounting for selection bias. Genome Biol. 2010, 11, 1-12. [CrossRef]

26. Mao, X.; Cai, T.; Olyarchuk, J.G.; Wei, L. Automated genome annotation and pathway identification using the KEGG Orthology (KO) as a controlled vocabulary. Bioinformatics 2005, 21, 3787-3793. [CrossRef]

27. Xie, L.; Li, F.; Zhang, S.; Zhang, H.; Qian, W.; Li, P.; Zhang, S.; Sun, R. Mining for Candidate Genes in an Introgression Line by Using RNA Sequencing: The Anthocyanin Overaccumulation Phenotype in Brassica. Front. Plant Sci. 2016, 7, 1245. [CrossRef]

28. Dai, Y.; Yuan, L.; Zhang, S.; Wang, J.; Xie, S.; Zhao, M.; Chen, G.; Sun, R.; Wang, C. Comprehensive Evaluation for Cold Tolerance in Wucai (Brassica campestris L.) by the Performance Index on an Absorption Basis (PIabs). Agronomy 2019, 9, 61. [CrossRef]

29. Chen, G.; Ye, X.; Zhang, S.; Zhu, S.; Yuan, L.; Hou, J.; Wang, C. Comparative Transcriptome Analysis between Fertile and CMS Flower Buds in Wucai (Brassica campestris L.). BMC Genom. 2018, 19, 908. [CrossRef]

30. Song, G.Q.; Chen, Q. Comparative transcriptome analysis of nonchilled, chilled, and late-pink bud reveals flowering pathway genes involved in chilling-mediated flowering in blueberry. BMC Plant Biol. 2018, 18, 98. [CrossRef]

31. Huang, J.; Liu, X.; Wang, J.; Lu, Y. Transcriptomic analysis of Asiatic lily in the process of vernalization via RNA-seq. Mol. Biol. Rep. 2014, 41, 3839-3852. [CrossRef] [PubMed]

32. Yang, M.; Zhu, L.; Xu, L.; Pan, C.; Liu, Y. Comparative transcriptomic analysis of the regulation of flowering in temperate and tropical lotus (Nelumbo nucifera) by RNA-Seq. Ann. Appl. Biol. 2014, 165, 73-95. [CrossRef]

33. Wang, X.; Wang, H.; Wang, J.; Sun, R.; Wu, J.; Liu, S.; Bai, Y.; Mun, J.; Bancroft, I.; Cheng, F. The genome of the mesopolyploid crop species Brassica rapa. Nat. Genet. 2011, 43, 1035-1039. [CrossRef] [PubMed]

34. Cai, C.; Wang, X.; Liu, B.; Wu, J.; Liang, J.; Cui, Y.; Cheng, F.; Wang, X. Brassica rapa Genome 2.0: A Reference Upgrade through Sequence Re-assembly and Gene Re-annotation. Mol. Plant 2016, 10, 649-651. [CrossRef] [PubMed] 
35. Sablowski, R. Flowering and determinacy in Arabidopsis. J. Exp Bot. 2007, 58, 899-907. [CrossRef] [PubMed]

36. Davis, S.J. Integrating hormones into the floral-transition pathway of Arabidopsis thaliana. Plant Cell Environ. 2009, 32, 1201-1210. [CrossRef]

37. Alabadi, D.; Blazquez, M.A.; Carbonell, J.; Ferrandiz, C.; Perezamador, M.A. Instructive roles for hormones in plant development. Int. J. Plant Dev. Biol. 2009, 53, 1597-1608. [CrossRef]

38. Tiwari, S.B.; Wang, X.J.; Guilfoyle, H.T.J. AUX/IAA Proteins Are Active Repressors, and Their Stability and Activity Are Modulated by Auxin. Plant Cell 2001, 13, 2809-2822. [CrossRef]

39. Dharmasiri, N.; Dharmasiri, S.; Estelle, M. The F-box protein TIR1 is an auxin receptor. Nature 2005, 435, 441-445. [CrossRef]

40. Hagen, G.; Guilfoyle, T.J. Auxin-responsive gene expression: Genes, promoters and regulatory factors. Plant Mol. Biol. 2002, 49, 373-385. [CrossRef]

41. Pierrejerome, E.; Moss, B.L.; Nemhauser, J.L. Tuning the auxin transcriptional response. J. Exp. Bot. 2013, 64, 2557-2563. [CrossRef]

42. Ariizumi, T.; Hauvermale, A.L.; Nelson, S.K.; Hanada, A.; Steber, C.M. Lifting della repression of arabidopsis seed germination by nonproteolytic gibberellin signaling. Plant Physiol. 2013, 162, 2125-2139. [CrossRef] [PubMed]

43. Benavente, L.M.; Alonso, J.M. Molecular mechanisms of ethylene signaling in Arabidopsis. Mol. BioSyst. 2006, 2, 165-173. [CrossRef] [PubMed]

44. Sakai, H.; Hua, J.; Chen, Q.G.; Chang, C.; Medrano, L.; Bleecker, A.B.; Meyerowitz, E.M. ETR2 is an ETR1-like gene involved in ethylene signaling in Arabidopsis. Proc. Natl. Acad. Sci. USA 1998, 95, 5812-5817. [CrossRef] [PubMed]

45. Clark, K.; Larsen, P.B.; Wang, X.; Chang, C. Association of the Arabidopsis CTR1 Raf-like kinase with the ETR1 and ERS ethylene receptors. Proc. Natl. Acad. Sci. USA 1998, 95, 5401-5406. [CrossRef]

46. Ouaked, F.; Rozhon, W.; Lecourieux, D.; Hirt, H. A MAPK pathway mediates ethylene signaling in plants. EMBO J. 2003, 22, 1282-1288. [CrossRef]

47. Ecker, J.R. Reentry of the Ethylene MPK6 Module. Plant Cell 2004, 16, 3169-3173. [CrossRef]

48. EIN2 and EIN3 in Ethylene Signalling. Available online: https://onlinelibrary.wiley.com/doi/pdf/10.1002/ 9781118223086.ch7 (accessed on 14 February 2012).

49. Chao, Q.; Rothenberg, M.; Solano, R.; Roman, G.; Terzaghi, W.; Ecker, J.R. Activation of the ethylene gas response pathway in Arabidopsis by the nuclear protein ETHYLENE-INSENSITIVE3 and related proteins. Cell 1997, 89, 1133-1144. [CrossRef]

50. Mccourt, P.; Creelman, R.A. The ABA receptors-We report you decide. Curr. Opin. Plant Biol. 2008, 11, 474-478. [CrossRef]

51. Ma, Y.; Szostkiewicz, I.; Korte, A.; Moes, D.; Yang, Y.; Christmann, A.; Grill, E. Regulators of PP2C Phosphatase Activity Function as Abscisic Acid Sensors. Science 2009, 324, 1064-1068.

52. Park, S.; Fung, P.; Nishimura, N.; Jensen, D.R.; Fujii, H.; Zhao, Y.; Lumba, S.; Santiago, J.; Rodrigues, A.; Chow, T.F.; et al. Abscisic Acid Inhibits Type 2C Protein Phosphatases via the PYR/PYL Family of START Proteins. Science 2009, 324, 1068-1071. [CrossRef] [PubMed]

53. Umezawa, T.; Sugiyama, N.; Mizoguchi, M.; Hayashi, S.; Myouga, F.; Yamaguchi-shinozaki, K.; Ishihama, Y.; Hirayama, T.; Shinozaki, K. Type 2C protein phosphatases directly regulate abscisic acid-activated protein kinases in Arabidopsis. Proc. Natl. Acad. Sci. USA 2009, 106, 17588-17593. [CrossRef] [PubMed]

54. Achard, P.; Cheng, H.; De Grauwe, L.; Decat, J.; Schoutteten, H.; Moritz, T.; Van Der Straeten, D.; Peng, J.; Harberd, N.P. Integration of Plant Responses to Environmentally Activated Phytohormonal Signals. Science 2006, 311, 91-94. [CrossRef]

55. Razem, F.A.; Elkereamy, A.; Abrams, S.R.; Hill, R.D. The RNA-binding protein FCA is an abscisic acid receptor. Nature 2006, 439, 290-294. [CrossRef] [PubMed]

56. Emilie, W.; Ekaterina, S.; Yann, A.; Laurence, M.; Thierry, H. Dynamics of Jasmonate Metabolism upon Flowering and across Leaf Stress Responses in Arabidopsis thaliana. Plants 2016, 5, 4.

57. Ferreira, F.J.; Kieber, J.J. Signaling: Cytokinin Signaling. Curr. Opin. Plant Biol. 2005, 8, 518-525. [CrossRef]

58. Loake, G.J.; Grant, M. Salicylic acid in plant defence-the players and protagonists. Curr. Opin. Plant Biol. 2007, 10, 466-472. [CrossRef] 
59. Staswick, P.E.; Tiryaki, I.; Rowe, M. Jasmonate Response Locus JAR1 and Several Related Arabidopsis Genes Encode Enzymes of the Firefly Luciferase Superfamily That Show Activity on Jasmonic, Salicylic, and Indole-3-Acetic Acids in an Assay for Adenylation. Plant Cell 2002, 14, 1405-1415. [CrossRef]

60. Diallo, A.; Agharbaoui, Z.; Badawi, M.A.; Alibenali, M.A.; Moheb, A.; Houde, M.; Sarhan, F. Transcriptome analysis of an mop mutant reveals important changes in global gene expression and a role for methyl jasmonate in vernalization and flowering in wheat. J. Exp. Bot. 2014, 65, 2271-2286. [CrossRef]

61. Daloia, M.; Bonhomme, D.; Bouche, F.; Tamseddak, K.; Ormenese, S.; Torti, S.; Coupland, G.; Périlleu, C. Cytokinin promotes flowering of Arabidopsis via transcriptional activation of the FT paralogue TSF. Plant J. 2011, 65, 972-979. [CrossRef]

62. Denay, G.; Chahtane, H.; Tichtinsky, G.; Parcy, F. A flower is born: An update on Arabidopsis floral meristem formation. Curr. Opin. Plant Biol. 2017, 35, 15-22. [CrossRef] [PubMed]

63. Genoud, T.; Buchala, A.J.; Chua, N.; Metraux, J. Phytochrome signalling modulates the SA-perceptive pathway in Arabidopsis. Plant J. 2002, 31, 87-95. [CrossRef] [PubMed]

64. Martínez, C.; Pons, E.; Prats, G.; León, J. Salicylic acid regulates flowering time and links defence responses and reproductive development. Plant J. 2003, 37, 209-217. [CrossRef] [PubMed]

65. Perilleux, C.; Bernier, G. Leaf carbohydrate status in Lolium temulentum during the induction of flowering. New Phytol. 1997, 135, 59-66. [CrossRef]

66. Arrom, L.; Munnebosch, S. Sucrose accelerates flower opening and delays senescence through a hormonal effect in cut lily flowers. Plant Sci. 2012, 188, 41-47. [CrossRef] [PubMed]

67. Sturm, A.; Tang, G. The sucrose-cleaving enzymes of plants are crucial for development, growth and carbon partitioning. Trends Plant Sci. 1999, 4, 401-407. [CrossRef]

68. Sawitri, W.D.; Afidah, S.N.; Nakagawa, A.; Hase, T.; Sugiharto, B. Identification of UDP-glucose binding site in glycosyltransferase domain of sucrose phosphate synthase from sugarcane (Saccharum officinarum) by structure-based site-directed mutagenesis. Biophys. Rev. 2018, 10, 293-298. [CrossRef]

69. Hedhly, A.; Vogler, H.; Schmid, M.W.; Pazmino, D.; Gagliardini, V.; Santelia, D.; Grossniklaus, U. Starch Turnover and Metabolism during Flower and Early Embryo Development. Plant Physiol. 2016, 172, 2388-2402. [CrossRef]

70. Dauvillee, D.; Chochois, V.; Steup, M.; Haebel, S.; Eckermann, N.; Ritte, G.; Ral, J.-P.; Colleoni, C.; Hicks, G.; Wattebled, F.; et al. Plastidial phosphorylase is required for normal starch synthesis in Chlamydomonas reinhardtii. Plant J. 2006, 48, 274-285. [CrossRef]

71. Wang, T.; Wright, D.; Xu, H.; Yang, Y.; Zheng, R.; Shi, J.; Chen, R.; Wang, L. Expression Patterns, Activities and Sugar Metabolism Regulation of Sucrose Phosphate Synthase, Sucrose Synthase, Neutral Invertase and Soluble Acid Invertase in Different Goji Cultivars during Fruit Development. Russ. J. Plant Phys. 2019, 66, 29-40. [CrossRef]

72. Niittyla, T.; Messerli, G.; Trevisan, M.; Chen, J.; Smith, A.M.; Zeeman, S.C. A Previously Unknown Maltose Transporter Essential for Starch Degradation in Leaves. Science 2004, 303, 87-89. [CrossRef] [PubMed]

73. Yue, C.; Cao, H.; Lin, H.; Hu, J.; Ye, Y.; Li, J.; Hao, Z.; Hao, X.; Sun, Y.; Yang, J.; et al. Expression patterns of alpha-amylase and beta-amylase genes provide insights into the molecular mechanisms underlying the responses of tea plants (Camellia sinensis) to stress and postharvest processing treatments. Planta 2019, 250, 281-298. [CrossRef] [PubMed]

74. Weise, S.E.; Weber, A.P.M.; Sharkey, T.D. Maltose is the major form of carbon exported from the chloroplast at night. Planta 2004, 218, 474-482. [CrossRef] [PubMed]

75. Van Dijken, A.; Schluepmann, H.; Smeekens, S. Arabidopsis Trehalose-6-Phosphate Synthase 1 Is Essential for Normal Vegetative Growth and Transition to Flowering. Plant Physiol. 2004, 135, 969-977. [CrossRef] [PubMed]

76. Gomez, L.D.; Gilday, A.D.; Feil, R.; Lunn, J.E.; Graham, I.A. AtTPS1-mediated trehalose 6-phosphate synthesis is essential for embryogenic and vegetative growth and responsiveness to ABA in germinating seeds and stomatal guard cells. Plant J. 2010, 64, 1-13. [CrossRef] [PubMed]

77. Paul, M.J.; Primavesi, L.F.; Jhurreea, D.; Zhang, Y. Trehalose Metabolism and Signaling. Ann. Rev. Plant Biol. 2008, 59, 417-441. [CrossRef]

78. Ponnu, J.; Wahl, V.; Schmid, M. Trehalose-6-phosphate: Connecting plant metabolism and development. Front. Plant Sci. 2011, 2, 70. [CrossRef] 
79. Wahl, V.; Ponnu, J.; Schlereth, A.; Arrivault, S.; Langenecker, T.; Franke, A.; Feil, R.; Lunn, J.E.; Stitt, M.; Schmid, M. Regulation of Flowering by Trehalose-6-Phosphate Signaling in Arabidopsis thaliana. Science 2013, 339, 704-707. [CrossRef]

80. Zhou, J.; Bai, Y.; Dai, R.; Guo, X.; Liu, Z.H.; Yuan, S. Improved Polysaccharide Production by Homologous Co-overexpression of Phosphoglucomutase and UDP Glucose Pyrophosphorylase Genes in the Mushroom Coprinopsis cinerea. J. Argic. Food Chem. 2018, 66, 4702-4709. [CrossRef]

81. Baron, D.; Bravo, J.P.; Maia, I.D.G.; Pina, A.; Ferreira, G. UGP gene expression and UDP-glucose pyrophosphorylase enzymatic activity in grafting annonaceous plants. Acta Physiol. Plant. 2016, 38, 1-8. [CrossRef]

82. Valverde, F.; Mouradov, A.; Soppe, W.J.; Ravenscroft, D.; Samach, A.; Coupland, G. Photoreceptor Regulation of CONSTANS Protein in Photoperiodic Flowering. Science 2004, 303, 1003-1006. [CrossRef] [PubMed]

83. Paina, C.; Byrne, S.; Domnisoru, C.; Asp, T. Vernalization mediated changes in the Lolium perenne transcriptome. PLoS ONE 2014, 9, e107365. [CrossRef] [PubMed]

84. Zheng, Y.; Luo, L.; Liu, Y.; Yang, Y.; Wang, C.; Kong, X.; Yang, Y. Effect of vernalization on tuberization and flowering in the Tibetan turnip is associated with changes in the expression of FLC homologues. Plant Divers. 2018, 40, 50-56. [CrossRef] [PubMed]

(C) 2020 by the authors. Licensee MDPI, Basel, Switzerland. This article is an open access article distributed under the terms and conditions of the Creative Commons Attribution (CC BY) license (http://creativecommons.org/licenses/by/4.0/). 